\title{
On the Design of Optimal TDM Schedules for Broadcast WDM Networks with Arbitrary Transceiver Tuning Latencies
}

\author{
George N. Rouskas Vijay Sivaraman
}

TR-95-07

June 1995

\begin{abstract}
We consider the problem of scheduling packet transmissions in a broadcast, single-hop WDM network. Tunability is provided only at one end, namely, at the transmitters. Our objective is to design schedules of minimum length to satisfy a set of traffic requirements given in the form of a demand matrix. We address a fairly general version of the problem as we allow arbitrary traffic demands and arbitrary transmitter tuning latencies. The contribution of our work is twofold. First we define a special class of schedules which permit an intuitive formulation of the scheduling problem. Based on this formulation we present algorithms which construct schedules of length equal to the lower bound provided that the traffic requirements satisfy certain optimality conditions. We also develop heuristics which, in the general case, give schedules of length equal or very close to the lower bound. Secondly, we identify two distinct regions of network operation. The first region is such that the schedule length is determined by the tuning requirements of transmitters; when the network operates within the second region however, the length of the schedule is determined by the traffic demands, not the tuning latency. The point at which the network switches between the two regions is identified in terms of system parameters such as the number of nodes and channels, and the tuning latency. Accordingly, we show that it is possible to appropriately dimension the network to offset the effects of even large values of the tuning latency.
\end{abstract}

\author{
Department of Computer Science \\ North Carolina State University \\ Raleigh, NC 27695-8206
}




\section{Introduction}

Wavelength division multiplexing (WDM) has been increasingly receiving attention as the most promising approach to fully exploiting the vast information-carrying capacity of single-mode fiber. By dividing the bandwidth of the optical medium into narrower channels, WDM makes it possible to design and implement communication networks with a large number of users, and an aggregate throughput that can be in the order of Terabits per second. In particular, our focus in this paper is on a WDM network architecture known as the single-hop architecture [1]. Single-hop networks are especially attractive as they are all-optical in nature. In other words, any information transmitted into the medium remains in the optical form until it reaches its destination, eliminating the so-called electro-optic bottleneck [2].

Critical to the design of single-hop networks is the availability of tunable lasers and/or optical filters, devices with the ability to tune across, and access, all available channels. Such devices do exist today; however, their capabilities are limited in terms of both tunability range and speed. Work in improving the performance characteristics of tunable devices proceeds at a fast pace; but

the ideal device, one that can tune across the useful optical spectrum in sub-microsecond times [3] remains elusive, and, barring a technological breakthrough, will remain so at least for the foreseeable future. We show, however, that careful network design can mask the effects of non-ideal devices, making it possible to build single-hop WDM networks using currently available tunable optical transceivers.

A typical WDM environment with a large number of users will have only a limited number of available wavelengths. Under such a scenario, it seems possible to mask the tuning times by letting a transceiver that was previously idle take the place of one that is taken off-line for retuning. Our work provides insight into this potentially difficult problem of coordinating the packet transmissions and retunings among the various nodes in the network. In addition to providing heuristics, as well as optimal (under certain conditions) scheduling algorithms, we will show that, if the network operates within a certain region, even large values of the tuning latency have no significant effect on the length of the schedule (which provides a measure of the delay and throughput performance). Our results are consistent with those in [4] which were derived under different traffic assumptions and for small values of tuning latency.

This paper is organized as follows. Section 2 contains some background, including discussion of related work on scheduling packet transmissions in WDM/TDM single-hop networks with tuning latency. In Section 3 we describe our system and traffic model, and formally introduce the concept of a schedule. In Section 4 we formulate the problem of finding schedules of minimum length, and 
show that it is $\mathcal{N P}$-complete; we also derive lower bounds on the schedule length, and discuss the effect of the dominant bound on the network operation. We introduce a special class of schedules in Section 5, and proceed to develop scheduling algorithms which, under certain conditions, construct optimal schedules within this class. Scheduling heuristics are developed in Section 6, and in Section 7 we present some numerical results. We then summarize our work, and conclude the paper in Section 8.

\section{Background}

In the recent past, significant effort has been devoted to the design and study of protocols for single-hop WDM networks. In general, the various design approaches take one of two directions, depending on their assumptions regarding the relative values of packet transmission time and transceiver tuning times. In order to characterize these approaches, let $\delta$ denote the normalized tuning latency, expressed in units of packet transmission time. The value of $\delta$ depends on the data rate, the packet size, and the transceiver tuning time, and can be less than, equal to, or greater than one.

Underlying the design of a broad class of architectures is the assumption that $\delta \ll 1$, i.e., that transceiver tuning times are negligible compared to the duration of a packet transmission. This assumption is reasonable for communication environments with data rates in the order of a few hundreds Megabits per second, and relatively large packet sizes. For instance, with a 155 Megabits per second rate, 6000 bit packets, and $1 \mu$ s tuning time, the normalized tuning latency $\delta \approx 0.026 \ll 1$. Accordingly, a padding equal to $\delta$ time units can be included within each slot to allow the transceivers sufficient time to switch between wavelengths, with minimal effects on the overall performance. This is reflected in the design of network architectures and protocols for such environments $[5,6,7,8,9,10]$ which has been geared towards improving the delay and throughput characteristics of the network under various traffic assumptions, completely ignoring transceiver tuning times.

With the current trend, however, being towards ever increasing data rates (Gigabits per second and beyond) and diminishing packet sizes (e.g., 53-byte ATM cells), emerging communication environments are such that the tuning times of even the fastest available tunable optical devices dominate over packet transmission times, making $\delta$ comparable to, and even greater than, 1 . Including a padding equal to $\delta$ within each slot would be highly inefficient in this case; instead, it is highly desirable to have the slot time equal to the packet transmission time alone. Let us now 
define $\Delta$ as

$$
\Delta=\lceil\delta\rceil
$$

Observe that, in a slotted system with a slot time equal to the packet transmission time, a transceiver instructed to switch to a new channel will be unavailable for a number of slots equal to $\Delta$. We will use the term tuning slots in future references to parameter $\Delta$.

Let $\Delta \geq 1$ be the number of tuning slots of the system under consideration. A straightforward approach to make the tuning latency transparent to higher level protocols, would be to equip each node with $\Delta+1$ transceivers. A node would then use transceiver $t, t=0, \ldots, \Delta$, in slots $t+$ $m(\Delta+1), m=0,1,2, \ldots$, only. This configuration would, in effect, appear to higher level protocols as a single transceiver that can tune infinitely fast between channels. Its obvious disadvantages, however, including the cost of hardware and the complexity of managing and coordinating packet transmissions/receptions from multiple transceivers, especially as $\Delta>1$, make this an unattractive solution. A design similar in concept, but oriented towards circuit switched traffic, can be found in [11].

If no extra hardware is used, minimizing the effects of transceiver tuning times on network performance is possible only through specially designed protocols. In [12], for instance, the TDMA scheme considered is such that the frame is divided into transmitting and tuning periods. Each transceiver operates on a fixed channel during a transmitting period; no transmissions take place during the tuning periods, which are reserved to retune transceivers to be ready for the next transmitting period. The objective is to minimize the number of tuning periods within the frame. The MaTPi protocol [13], on the other hand, is a reservation based protocol that can be used by stations to reserve, in real time, a time slot that is $\Delta$ slots in the future.

Another design approach, which we pursue in this work, is based on the observation that, when the number of stations, $N$, in the network is greater than the number of available wavelengths, $C$, at most $C$ stations may be transmitting at any given slot. The remaining stations may use that slot for retuning to a new channel, so that they will be ready to access that channel at a later slot. Thus, transceiver tuning times may (at least partially) be overlapped with transmissions by other stations, keeping channel utilization at high levels. The objective, then, is to design schedules of minimum length, given a traffic demand matrix. In $[14,15]$ uniform traffic demands are considered, and lower and upper bounds on the length of an optimal schedule are derived. The work in [4] considers a traffic demand matrix of 1's and 0's (representing the existence or not, respectively, of a head-of-line packet at the various queues), and values of $\delta \leq 1$. The main contribution of [4] was to identify, in terms of system parameters $N, C$, and $\delta \leq 1$, a region of operation for the network such that the inefficiency due to the tuning latency can be completely eliminated through 
a simple scheduling algorithm. Our work is more general, as it considers arbitrary traffic demands and arbitrary values of $\delta$; furthermore, we develop scheduling algorithms which guarantee that, within certain regions of operation, the tuning latency has no effect on network performance, thus extending the results of [4] to values of $\delta>1$.

The problem of scheduling non-uniform traffic under arbitrary tuning latencies has been previously studied in [16], where a scheduling heuristic was presented and shown to produce good results. There are significant differences between the work in [16] and ours, however. In contrast to [16] where one heuristic is used throughout, we make the fundamental observation that, depending on the traffic matrix and system parameters $N, C$, and $\delta$, the network can be operating in one of two distinct regions. We then develop two scheduling algorithms, one for each region, which we prove to be optimal under certain conditions; further, we demonstrate that an algorithm optimal for one region performs sub-optimally when applied to a network operating in the other region. We also present heuristics (again one for each region) that are quite different than the one in [16], and which are based on the intuition provided by an appropriate formulation of the scheduling problem.

\section{System Model}

We consider packet transmissions in an all-optical, single-hop WDM network with a passive star physical topology. Each of the $N$ nodes in the network employs one transmitter and one receiver. The passive star supports $C$ wavelengths, or channels ${ }^{1}, \lambda_{1}, \ldots, \lambda_{C}$. In general, $C \leq N$. Without loss of generality, we only consider tunable-transmitter, fixed-receiver (TT-FR) networks; all of our results can be easily adapted to fixed-transmitter, tunable-receiver systems.

Each tunable transmitter can be tuned to, and transmit on, any and all wavelengths $\lambda_{c}, c=$ $1, \ldots, C$. The fixed receiver at station $j$, on the other hand, is assigned wavelength $\lambda(j) \in$ $\left\{\lambda_{1}, \ldots, \lambda_{C}\right\}$. If the number of channels, $C$, is equal to the number of nodes, $N$, then each receiver is assigned a unique wavelength. When $C<N$, however, a single wavelength may be assigned to a number of receivers. We define $\mathcal{R}_{c}$ as the set of receivers sharing wavelength $\lambda_{c}$ :

$$
\mathcal{R}_{c}=\left\{j \mid \lambda(j)=\lambda_{c}\right\}, \quad c=1, \ldots, C
$$

Under the packet transmission scenario we are considering, there is an $N \times N$ traffic demand matrix $\mathbf{D}=\left[d_{i j}\right]$, with $d_{i j}$ representing the number of slots to be allocated for transmissions from source $i$ to destination $j$. Since a transmission on wavelength $\lambda_{c}$ is heard by all receivers listening

\footnotetext{
${ }^{1}$ The terms "wavelength" and "channel" will be used interchangeably throughout this paper.
} 
on $\lambda_{c}$, given a partition of the receiver set into sets $\mathcal{R}_{c}$, we obtain the collapsed $N \times C$ traffic matrix $\mathbf{A}=\left[a_{i c}\right]$. Element $a_{i c}$ of the collapsed matrix represents the number of slots to be assigned to source $i$ for transmissions on channel $\lambda_{c}$ :

$$
a_{i c}=\sum_{j \in \mathcal{R}_{c}} d_{i j}, \quad i=1, \ldots, N, c=1, \ldots, C
$$

Without loss of generality, we assume that $a_{i c}>0 \forall i, c$, that is, each source $i$ has to be allocated at least one slot on each channel ${ }^{2}$. We also let $D$ denote the total traffic demand, across all source-destination pairs:

$$
D=\sum_{i=1}^{N} \sum_{j=1}^{N} d_{i j}=\sum_{i=1}^{N} \sum_{c=1}^{C} a_{i c}
$$

There are several situations in which such a transmission scenario arises. For instance, under a gated service discipline, quantity $d_{i j}$ may represent the number of packets with destination $j$ in the queue of station $i$ at the moment the "gate" is closed. Alternatively, it may represent the number of slots to be allocated to the $(i, j)$ source-destination pair to meet certain quality of service (QOS) criteria; in the latter case $d_{i j}$ may not directly depend on actual queue lengths, but may be derived based on assumptions regarding the arrival process at the source. The exact nature of $d_{i j}$ is not important in this work and does not affect our conclusions, therefore, it will be left unspecified.

Finally, observe that, while the traffic matrix, D, is given, the collapsed matrix, $\mathbf{A}$, is not uniquely specified, but depends on the assignment of receivers to wavelengths. For the moment, we will assume that the receiver sets $\mathcal{R}_{c}$ are known; how to construct these sets will be discussed in Section 4.2.

\subsection{Transmission Schedules}

In the WDM environment we are considering, a simultaneous transmission by two or more stations on the same channel results in a collision. To avoid packet loss due to collisions, some form of coordination among transmitting sources is necessary [3]. A transmission schedule is an assignment of slots to source-channel pairs that provides this coordination: if slot $\tau$ is assigned to pair $\left(i, \lambda_{c}\right)$, then in slot $\tau$, source $i$ may transmit a packet to any of the receivers listening on wavelength $\lambda_{c}$. Exactly $a_{i c}$ slots must be assigned to the source-channel pair $\left(i, \lambda_{c}\right)$, as specified by the collapsed matrix A. However, this assignment is complicated by the fact that transmitters need time to tune from one wavelength to another.

\footnotetext{
${ }^{2}$ This assumption is reasonable, especially when the number of nodes, $N$, is significantly greater than the number of available channels (a likely scenario in WDM environments), as each channel will be shared by many receivers.
} 
If the $a_{i c}$ slots are contiguously allocated for all pairs $\left(i, \lambda_{c}\right)$, the schedule is said to be nonpreemptive; otherwise we have a preemptive schedule. Under a non-preemptive schedule, each transmitter will tune to each channel exactly once, minimizing the overall time spent for tuning. Since our objective is to assign slots so as to minimize the time needed to satisfy the traffic demands specified by the collapsed traffic matrix. A, we only consider non-preemptive schedules.

Formally, a non-preemptive schedule is defined as a set $\mathcal{S}=\left\{\tau_{i c}\right\}$, with $\tau_{i c}$ the first of a block of $a_{i c}$ contiguous slots assigned to the source-channel pair $\left(i, \lambda_{c}\right)$. Since each source has exactly one laser which needs $\Delta$ slots to tune between channels, all time intervals $\left[\tau_{i c}-1, \tau_{i c}+a_{i c}+\Delta-1\right.$ ) must be disjoint ${ }^{3}$, yielding a set of hardware constraints on schedule $\mathcal{S}$ :

$$
\left[\tau_{i c}-1, \tau_{i c}+a_{i c}+\Delta-1\right) \bigcap\left[\tau_{i c^{\prime}}-1, \tau_{i c^{\prime}}+a_{i c^{\prime}}+\Delta-1\right)=\phi \quad \forall c \neq c^{\prime}, \quad i=1, \ldots, N
$$

In addition, to avoid collisions, at most one transmitter should be allowed to transmit on a given channel in any given slot, resulting in a set of no-collision constraints:

$$
\left[\tau_{i c}-1, \tau_{i c}+a_{i c}-1\right) \bigcap\left[\tau_{i^{\prime} c}-1, \tau_{i^{\prime} c}+a_{i^{\prime} c}-1\right)=\phi \quad \forall i \neq i^{\prime}, \quad c=1, \ldots, C
$$

A non-preemptive schedule $\mathcal{S}$ is admissible if and only if $\mathcal{S}$ satisfies both the hardware and the no-collision constraints.

Consider now transmitter $i$ and an admissible schedule $\mathcal{S}=\left\{\tau_{i c}\right\}$. Based on the above discussion, transmitter $i$ can be in one of three states during a slot $\tau$.

1. Transmitting state, if, according to the schedule, $i$ is assigned to transmit on some channel $\lambda_{c}$. Transmitter $i$ is in the transmitting state in slots $\tau_{i c}$ through $\tau_{i c}+a_{i c}-1, c=1, \ldots, C$.

2. Tuning state. Immediately after completing its transmission on channel $\lambda_{c}, i$ instructs its laser to tune to the next channel, say, channel $\lambda_{c^{\prime}}$, and will be in the tuning state for exactly $\Delta$ slots.

3. Idle state. The laser at station $i$ will be ready to transmit on channel $\lambda_{c^{\prime}}$ at the beginning of slot $\tau=\tau_{i c}+a_{i c}+\Delta$. If, however, $\tau_{i c^{\prime}}>\tau$, $i$ will simply wait for slot $\tau_{i c^{\prime}}$ before it starts transmitting on channel $\lambda_{c^{\prime}}$. We say that $i$ is idle in these $\tau_{i c^{\prime}}-\tau$ slots.

Similarly, we say that channel $\lambda_{c}$ is busy in slot $\tau$ if some station has been assigned to transmit on $\lambda_{c}$ in that slot (because of the no-collision constraint, there will be exactly one such station), and idle, otherwise. Channel idling results in wasted bandwidth; one of the contributions of this work is to show that it is possible to properly dimension the network to minimize channel idling.

\footnotetext{
${ }^{3}$ We make the assumption that slot $\tau$ starts at time $\tau-1$ and occupies the time interval $[\tau-1, \tau)$.
} 

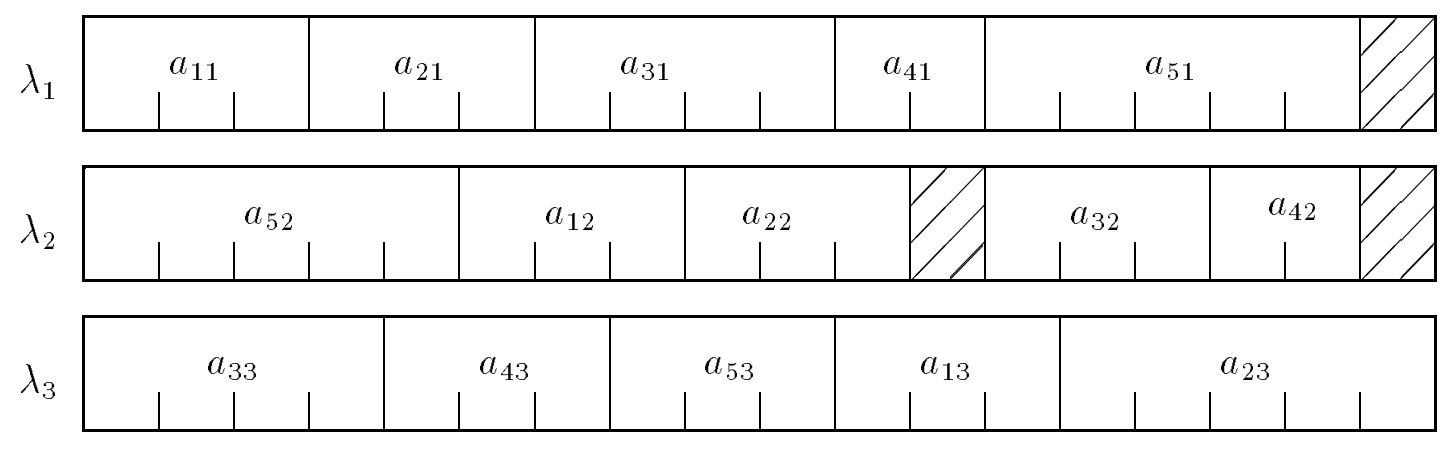

$\begin{array}{lllllllllllllllllll}\text { slot } & 1 & 2 & 3 & 4 & 5 & 6 & 7 & 8 & 9 & 10 & 11 & 12 & 13 & 14 & 15 & 16 & 17 & 18\end{array}$

Figure 1: An optimum length schedule for a network with $N=5, C=3$, and $\Delta=2$.

The length, $M$, of a schedule $\mathcal{S}$ for the collapsed traffic matrix $\mathrm{A}$ is the number of slots required to satisfy all traffic demands $a_{i c}$ under $\mathcal{S}$. An optimum length schedule for $\mathbf{A}$ is one with the least length among all schedules. Note that an optimum length schedule does not preclude the existence of slots with idle channels (see also Figure 1), but a schedule in which no channel is ever idle is necessarily an optimum length schedule.

Figure 1 shows an optimum length non-preemptive schedule for a network with $N=5$ nodes, $C=3$ channels, and $\Delta=2$; the collapsed traffic matrix $\mathbf{A}$ can be easily deduced from the figure. Observe that all hardware and no-collision constraints are satisfied. In particular, the first slot assigned to station 3 on channel $\lambda_{2}$ is slot 13, rather than slot 12, as its laser needs two slots to tune from $\lambda_{1}$ to $\lambda_{2}$. Also, the fact that channel $\lambda_{2}$ is idle in slots 12 and 18, and channel $\lambda_{1}$ is idle in slot 18 , does not affect the overall length of the schedule.

In the following, we make the assumption that the schedule repeats over time; in other words, if $\tau_{i c}$ is the start slot of transmitter $i$ on channel $\lambda_{c}$ under schedule $\mathcal{S}$ of length $M$, then so are slots $\tau_{i c}+k M, k=1,2,3, \ldots$, where $k$ denotes the $k$-th identical copy of the schedule as it repeats in time. If the traffic parameters $d_{i j}$ are derived based on the behavior and required quality of service of longer term (relative to a packet transmission time) connections between the various source-destination pairs, we expect the schedule to repeat until a change in traffic demands triggers an update of the demand matrix. Under the gated service discipline scenario discussed above, however, a new schedule has to be computed after all transmissions under the current schedule have been completed. We now argue that the schedules we derive are applicable even under the latter scenario.

If the schedule is used only once, then a period of $\Delta$ tuning slots is necessary to allow transmitters to tune to their initial channels; no transmissions are possible during this tuning period. On 
the other hand, if the schedule repeats over time, this tuning period can be overlapped with transmissions in the previous frame of the schedule, possibly resulting in a smaller overall schedule length ${ }^{4}$. In any case, the length of a schedule derived under the assumption that transmissions repeat over time will be at most $\Delta$ slots smaller than if this assumption is not made. We can then use the schedules derived here in situations where a schedule is used only once, after adding an initial period of $\Delta$ slots. Furthermore, even though our assumption does affect the schedule length somewhat, it does not affect our conclusions about the network's regions of operation, to be discussed shortly.

Unless otherwise specified, from now on the term "schedule" will be used as an abbreviation for "admissible non-preemptive schedule".

\section{Schedule Optimization and Lower Bounds}

The length, $M$, of a schedule for a traffic matrix $\mathbf{D}$, is a measure of both the packet delay incurred while transmitting $\mathbf{D}$, and the system-wide throughput (the average number of packets transmitted per slot, $\frac{D}{M}$ ). Our objective then, is to determine an optimum length schedule to transmit the demand matrix $\mathbf{D}$, as such a schedule would both minimize the delay and maximize throughput. This problem, which we will call the Packet Scheduling with Tuning Latencies (PSTL) problem, can be stated concisely as:

Problem 4.1 (PSTL) Given the number of nodes, $N$, the number of available wavelengths, $C$, the traffic demand matrix, $\mathbf{D}=\left[d_{i j}\right]$, and the tuning slots, $\Delta$, find a schedule of minimum length for matrix $\mathbf{D}$.

Problem PSTL can be logically decomposed into two subproblems:

- the sets of receivers, $\mathcal{R}_{c}$, sharing wavelength $\lambda_{c}, c=1, \ldots, C$, must be obtained, and from them the collapsed traffic matrix, $\mathbf{A}=\left[a_{i c}\right]$, constructed, and

- for all $i$ and $c$, a way of placing the $a_{i c}$ slots to minimize the length of the schedule must be determined.

Let us now turn our attention to the second subproblem; for reasons that will become apparent shortly, we will refer to this as the Open-Shop Scheduling with Tuning Latencies (OSTL) problem. It can be expressed formally as a decision problem:

\footnotetext{
${ }^{4}$ Actually, a tuning period of $\Delta$ slots is still needed the very first time the schedule is used, but it can be ignored, especially if the schedule repeats for a relatively large number of times.
} 
Problem 4.2 (OSTL) Given the number of nodes, $N$, the number of available wavelengths, $C$, the collapsed traffic demand matrix, $\mathbf{A}=\left[a_{i c}\right]$, the tuning slots, $\Delta \geq 0$, and an overall deadline, $M>0$, is there a schedule $\mathcal{S}=\left\{\tau_{i c}\right\}$ that meets the deadline, in other words, is there a schedule of length at most $M$, satisfying constraints (5) and (6)?

As stated, $O S T L$ is a generalization of the non-preemptive open-shop scheduling $(O S)$ problem studied in $[17]^{5}$; it reduces to the latter when we let $\Delta=0$. It was shown in [17] that problem $O S$ is $\mathcal{N} \mathcal{P}$-complete when the number of wavelengths is $C \geq 3$. But for $C=2$, problem $O S$ admits a polynomial-time solution, and algorithm $O P E N_{-} S H O P$ was developed in [17] that constructs an optimum length $O S$ schedule in time linear in the number of nodes, $N$.

Drawing upon the results of [17], we now prove the following theorem, which confirms our intuition that $O S T L$ is in a sense more difficult than $O S$. Furthermore, it implies that a polynomialtime algorithm for $O S T L$, and consequently for PSTL, is unlikely to be found.

Theorem 4.1 OSTL is $\mathcal{N} \mathcal{P}$-complete for any fixed $C \geq 2$.

Proof. See Appendix A.

We now derive lower bounds for problems PSTL and OSTL, and discuss their implications.

\subsection{Lower Bounds for PSTL and OSTL}

First, observe that the length of any schedule cannot be smaller than the number of slots required to satisfy all transmissions on any given channel, yielding the bandwidth bound:

$$
M_{b w}^{(l)}=\max _{1 \leq c \leq C}\left\{\sum_{i=1}^{N} a_{i c}\right\} \geq \frac{D}{C}
$$

Note that the term in the brackets depends on the assignment of receive wavelengths to the nodes (i.e., the sets $\mathcal{R}_{c}$ ); the rightmost term, however, depends only on the total traffic demand, $D$, and is a lower bound on PSTL independently of the actual elements $d_{i j}$ of the demand matrix D. Expression (7) implies that, given the number of wavelengths (which determines the amount of bandwidth available), the bandwidth bound is minimized when the traffic load is perfectly balanced across the $C$ channels.

\footnotetext{
${ }^{5}$ In the terminology of [17], $C$ is the number of processors and $N$ is the number of jobs. Each job consists of $C$ tasks; the $c$-th task, $c=1, \ldots, C$, of job $i$ requires $a_{i c}$ processing time, and is to be processed by processor $c$.
} 
We can obtain a different lower bound by adopting a transmitter's point of view. Each transmitter $i$ needs a number of slots equal to the number of packets it has to transmit plus the number of slots required to tune to each of $C$ wavelengths ${ }^{6}$. We call this the tuning bound:

$$
M_{t}^{(l)}=\max _{1 \leq i \leq N}\left\{\sum_{c=1}^{C} a_{i c}\right\}+C \Delta=\max _{1 \leq i \leq N}\left\{\sum_{j=1}^{N} d_{i j}\right\}+C \Delta \geq \frac{D}{N}+C \Delta
$$

The tuning bound is independent of the assignment of receive wavelengths to the nodes, and only depends on the system parameters $N, C$, and $\Delta$, and the total traffic demand $D$; it is minimized when each source contributes equally to the total traffic demand. We now obtain the overall lower bound as

$$
M^{(l)}=\max \left\{M_{b w}^{(l)}, M_{t}^{(l)}\right\}
$$

This overall bound is minimized when

$$
\frac{D}{C}=\frac{D}{N}+C \Delta \Leftrightarrow \frac{D}{C}=\frac{N C \Delta}{N-C}
$$

It is interesting to note that the quantity $\frac{N C \Delta}{N-C}$ is independent of the demand matrix, and as such it characterizes the network under consideration. We will call this quantity the critical length. Now, the relationship (10) between the minimum bandwidth bound, $\frac{D}{C}$, and the critical length is a fundamental one, and represents the point at which wavelength concurrency balances the tuning latency. Indeed, if a schedule has length equal to the critical length, because of (10) it is such that exactly $C$ (respectively, $N-C$ ) nodes are in the transmitting (respectively, tuning) state within each slot. Consequently, all $N C \Delta$ tuning slots are overlapped with packet transmissions, and vice versa. Such a schedule is highly desirable, as it has three important properties: (a) it completely masks the tuning latency, (b) it is the shortest schedule for transmitting a total demand of $D$ packets, and (c) it achieves $100 \%$ utilization of the available bandwidth, as no channel is ever idle.

The significance of the actual schedule length relative to the critical length is explored in the following subsection.

\subsection{Bandwidth Limited vs. Tuning Limited Networks}

To get further insight on (10), let us consider the case of uniform traffic, whereby each source has $\beta \geq 1$ packets for each possible destination:

$$
\left.d_{i j}=\beta \geq 1 \quad \forall i, j \Rightarrow D=\beta N^{2} \quad \text { (integer } \beta\right)
$$

\footnotetext{
${ }^{6}$ Recall that we have assumed that $a_{i c}>0$ for all $i$ and $c$.
} 
This is a generalization of the all-to-all schedules studied in $[14,4]$, where the value of $\beta$ was taken equal to 1 . Substituting this value of $D$ into (10) we get

$$
\frac{\beta N^{2}}{C}=\beta N+C \Delta \Leftrightarrow \frac{\beta N^{2}}{C}=\frac{N C \Delta}{N-C}
$$

In $[14,4]$ the quadratic equation (12) was solved (with $\beta=1$ ) to obtain the value of $C$ that minimizes the lower bound for all-to-all schedules. Typically, however, $C, N$, and $\Delta$ are given parameters; one could then solve (12) to obtain an optimal value for $\beta$, which we will denote with $\beta^{\star}$; in general, $\beta^{\star}$ may not be an integer.

$$
\beta^{\star}=\frac{C^{2}}{N(N-C)} \Delta
$$

Suppose now that we choose $\beta<\beta^{\star}$ in (11); for simplicity, also let $N=k C$, so that the traffic demand can be perfectly balanced across the channels. In this case, the tuning bound $\beta N+C \Delta$ becomes greater than the bandwidth bound $\frac{\beta N^{2}}{C}$, and the length of the schedule is determined by the transmitter tuning requirements ${ }^{7}$. Since the total traffic demand is $\beta N^{2}$ and $\beta<\beta^{\star}$, the throughput achievable under such a schedule is

$$
\frac{\beta N^{2}}{\beta N+C \Delta}<C
$$

As we can see, the larger the value of $\beta$ the higher the throughput; once the value of $\beta$ has increased beyond $\beta^{\star}$, the bandwidth bound becomes dominant and the throughput becomes equal to its maximum value, $C$.

Increasing the value of $\beta$, however, has the effect of increasing the length of the schedule, either through the tuning bound $\beta N+C \Delta$, or through the bandwidth bound $\frac{\beta N^{2}}{C}$. But this length is a measure of packet delay, and cannot be increased beyond a certain level perceived as acceptable by the various higher layer applications. Within the family of matrices described in (11) therefore, the demand matrix corresponding to the value $\beta=\left\lceil\beta^{\star}\right\rceil$ achieves a perfect balance between delay and throughput, as it provides for the smallest schedule length that results in a $100 \%$ channel utilization. One might have to settle for less than $100 \%$ utilization, however, if satisfying the delay requirements would mean choosing $\beta<\left\lceil\beta^{\star}\right\rceil$. It is in these situations that advances in optical device technology would really make a difference ${ }^{8}$. From (13) we see that the value of $\beta^{\star}$, and consequently, the value of the critical length, is proportional to $\Delta$. Employing faster tunable transceivers would then bring

\footnotetext{
${ }^{7}$ As we shall see in the following section, when $C$ is a divisor of $N$, it is always possible to construct an optimal schedule (in this case, a schedule of length equal to the tuning bound) for uniform traffic demands.

${ }^{8}$ In contrast, the conclusion in [4] was that further advances in device technology would have negligible impact. This conclusion however, was due to the fact that only the case $\Delta=1$ was considered there.
} 
$\beta^{\star}$ closer to the acceptable (in terms of delay) operating value of $\beta$, and improve the throughput (see also (14)). Alternatively, according to (13), the same effect could be achieved by employing fewer wavelengths, a larger number of nodes, or a combination of the two.

The above observations are of general nature, applying to non-uniform demand matrices as well. In general, we will say that a network is

- tuning limited, if the tuning bound dominates, i.e., $M^{(l)}=M_{t}^{(l)}>M_{b w}^{(l)}$, or

- bandwidth limited, if the bandwidth bound is dominant; then, $M^{(l)}=M_{b w}^{(l)}>M_{t}^{(l)}$.

To see why this distinction is important, note that any near-optimal scheduling algorithm, including the ones to be presented shortly, will construct schedules of length very close to the lower bound. If the network is tuning limited, the length of the schedule is determined by the tuning bound in (8), which in turn is directly affected by the tuning latency. The schedule length of a bandwidth limited network, on the other hand, depends only on the traffic requirements of the dominant channel, i.e., the channel $\lambda_{c}$ such that $\sum_{i=1}^{N} a_{i c}=M_{b w}^{(l)}$.

Based on this discussion, it is desirable to operate the network at the bandwidth limited region, as doing so would eliminate the effects of tuning latency. For uniform traffic we saw that this can be accomplished by selecting $\beta=\left\lceil\beta^{\star}\right\rceil$. But the effect of choosing such a value for $\beta$ is to make the bandwidth bound greater than the critical length in (12). In the general case (non-uniform traffic matrix $\mathbf{D})$ we would like to make the bandwidth bound in (10) greater than the critical length:

$$
\frac{D}{C}>\frac{N C \Delta}{N-C}
$$

Given a value for $\Delta$, and some information about the delay requirements of higher layer applications, expression (15) may be satisfied by carefully dimensioning the network (i.e., initially choosing appropriate values for $N$ and $C$ ) so that it operates in the bandwidth limited region. Since, however, delay constraints and/or constraints on the values of $N$ and $C$ may make it impossible to satisfy (15) for a given system, in the following we develop scheduling algorithms and heuristics for both regions of network operation.

Let us now suppose that expression (15) is satisfied, i.e., that the network operates in the bandwidth limited region with the bandwidth bound $M_{b w}^{(l)}$ the dominant one. Recall that $M^{(l)}$ represents the total slot requirements for some channel, hence, under the non-uniform traffic scenario we are considering, it is possible for $M^{(l)}$ to be significantly greater than $\frac{D}{C}$. Since, assuming that a nearoptimal algorithm is available, the length of the final schedule will depend on $M^{(l)}$, it is extremely important that the receiver sets $\mathcal{R}_{c}$ be constructed so that the offered traffic is well balanced across 
all channels ${ }^{9}$. This load balancing problem [18] is a well-known and widely-studied $\mathcal{N} \mathcal{P}$-complete problem (refer also to the PARTITION problem in Appendix A), and several heuristics (such as the one in [19] which guarantees a performance of at most 1.22 times away from the optimal) as well as polynomial approximation algorithms have been derived for it. As such, we will not consider this problem any further, but we will once more emphasize the importance of using some approximation scheme to effectively balance the traffic across the channels, in addition to the heuristics presented here for the $O S T L$ problem.

\section{A Class of Schedules for $O S T L$}

Let $\mathrm{A}$ be a collapsed traffic matrix, and $\mathcal{S}$ a schedule of length $M$ satisfying the hardware and no-collision constraints (5) and (6), respectively. Consider now the order in which the various transmitters are assigned slots within, say, channel $\lambda_{1}$, starting with some transmitter $\pi_{1}$. We will say that $s_{1}=\left(\pi_{1}, \pi_{2}, \ldots, \pi_{N}\right)$ is the transmitter sequence on channel $\lambda_{1}$ if $\pi_{2}$ is the first node after $\pi_{1}$ to transmit on $\lambda_{1}, \pi_{3}$ is the second such node, and so on. Since we have assumed that schedule $\mathcal{S}$ repeats over time, after node $\pi_{N}$ has transmitted its packets on $\lambda_{1}$, the sequence of transmissions implied by $s_{1}$ above starts anew ${ }^{10}$. Similarly, we will say that $v_{1}=\left(\lambda_{\pi_{1}}, \lambda_{\pi_{2}}, \ldots, \lambda_{\pi_{C}}\right)$ is the channel sequence for node 1 , if this is the order in which node 1 is assigned to transmit on the various channels, starting with channel $\lambda_{\pi_{1}}$.

Given $\mathcal{S}$, the transmitter sequences with $\pi_{1}$ as the first node, are completely specified for all channels $\lambda_{c}$. In general, these sequences can be different for the various channels. However, in what follows we concentrate on a class of schedules such that the transmitter sequences (with $\pi_{1}$ as the first node) are the same for all channels:

$$
s_{c}=\left(\pi_{1}, \pi_{2}, \ldots, \pi_{N}\right) \quad c=1, \ldots, C
$$

It is easy to see that the class of schedules defined in (16) is equivalent to the class of schedules such that the channel sequences (with $\lambda_{\pi_{1}}$ as the first channel) are the same for all nodes ${ }^{11}$ :

$$
v_{i}=\left(\lambda_{\pi_{1}}, \lambda_{\pi_{2}}, \ldots, \lambda_{\pi_{C}}\right) \quad i=1, \ldots, N
$$

\footnotetext{
${ }^{9}$ Recall that constructing the sets $\mathcal{R}_{c}$ was the first of two subproblems into which problem PSTL was decomposed; the second being, of course, problem $O S T L$.

${ }^{10}$ Note that, since the schedule repeats over time, any contiguous chunk of $M$ slots constitutes a frame. Furthermore, frames on the various channels starting with the transmissions of, say, node $\pi_{1}$, will not be aligned in time (refer also to Figure 2).

${ }^{11}$ By "equivalent" we mean that if a schedule is such that the transmitter sequence is the same for all channels, then the channel sequence is the same for all transmitters, and vice versa.
} 


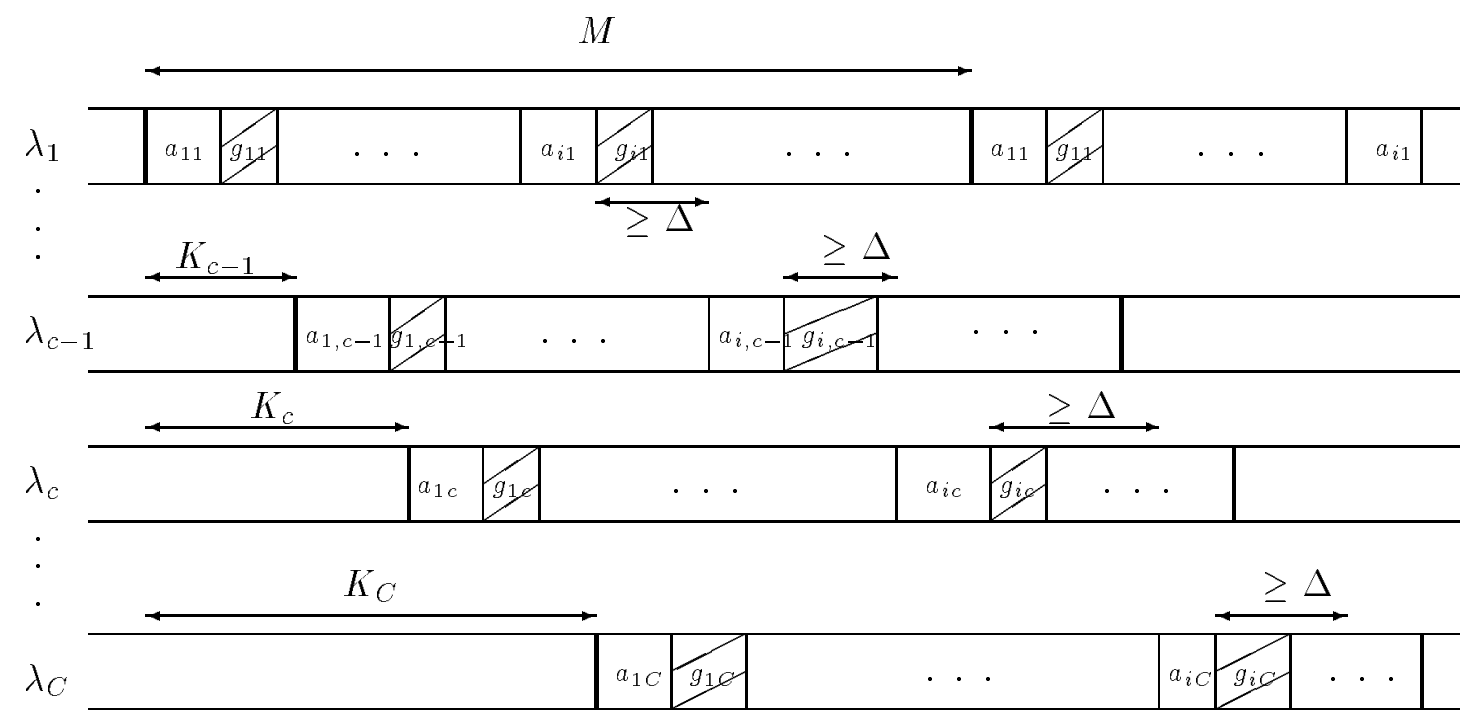

Figure 2: Schedule for a bandwidth limited network

Our examination of this class of schedules is motivated by several factors. First, the OPEN_SHOP algorithm for the $O S$ problem with $C=2$ channels [17] produces optimal schedules within this class. Secondly, for a uniform collapsed traffic matrix (i.e., $a_{i c}=a \forall i, c$ ), optimal schedules within this class do exist for the $O S T L$ problem. More importantly, this class of schedules greatly simplifies the analysis, allowing us to formulate the OSTL problem in a way that provides insight into the properties of good scheduling algorithms. As a result, for schedules in this class, we have been able to prove certain optimality properties and derive scheduling algorithms, and have obtained optimal or near-optimal schedules for a wide range of the system parameters $N, C$, and $\Delta$.

We now proceed to derive sufficient conditions for optimality, as well as algorithms for constructing optimal schedules within the class of schedules defined in (16) and (17). In our study, we distinguish between bandwidth limited and tuning limited networks. As we shall shortly show, different conditions of optimality apply to each of the two cases; thus, scheduling algorithms specially designed for bandwidth limited networks perform sub-optimally on tuning limited networks, and vice versa.

\subsection{Bandwidth Limited Networks}

We start by presenting an alternative formulation of problem OSTL, applicable to bandwidth limited schedules within the class (16). This new formulation will provide insight into the design of good scheduling algorithms. 
Let $\mathcal{S}$ be a schedule of length $M$ for a bandwidth limited network, and let $(1,2, \ldots, N)$ be the transmitter sequence on all channels. For each channel, consider the frame which begins with the first slot assigned to transmitter 1 . Let the start of the frame on channel $\lambda_{1}$ be our reference point, and let $K_{c}$ denote the distance, in slots, between the start of a frame on channel $\lambda_{c}$ and the start of the frame on the first channel; this is illustrated in Figure 2. Note also that $K_{1}=0$.

Consider now the transmissions on, say, channel $\lambda_{c}$, within a frame of $M$ slots. Following the $a_{1 c}$ slots assigned to transmitter 1 , the next $a_{2 c}$ slots are assigned to transmitter 2, unless this assignment does not allow the laser of 2 enough time to tune from $\lambda_{c-1}$ to $\lambda_{c}$. In the latter case, channel $\lambda_{c}$ has to remain idle for a number of slots before node 2 starts transmitting. In general, we will let $g_{i c}$ denote the number of slots that channel $\lambda_{c}$ remains idle between the end of transmissions by node $i$ and the start of transmissions by node $i+1$; we will refer to quantities $g_{i c}$ as the gaps within the channels.

Based on the above discussion, the problem of finding an optimum length schedule such that (a) the schedule is within the class defined in (16) and (b) the transmitter sequence is $(1,2, \ldots, N)$, can be formulated as an integer programming problem, to be referred to as bandwidth limited OSTL (BW-OSTL), as follows.

$$
B W-O S T L: \quad \min _{g_{i c}, K_{c}} M=\max _{c}\left\{\sum_{i=1}^{N}\left(a_{i c}+g_{i c}\right)\right\}
$$

subject to:

$$
\begin{gathered}
K_{c}+\sum_{j=1}^{i-1}\left(a_{j c}+g_{j c}\right) \geq K_{c-1}+\sum_{j=1}^{i-1}\left(a_{j, c-1}+g_{j, c-1}\right)+a_{i, c-1}+\Delta \quad c=2, \ldots, C, i=1, \ldots, N \\
M+\sum_{j=1}^{i-1}\left(a_{j 1}+g_{j 1}\right) \geq K_{C}+\sum_{j=1}^{i-1}\left(a_{j C}+g_{j C}\right)+a_{i C}+\Delta \quad i=1, \ldots, N \\
g_{i c}, K_{c}, M: \text { integers } ; g_{i c} \geq 0 \forall i, c ; K_{1}=0 ; K_{c}>K_{c-1} c=2, \ldots, C ; M>K_{C}
\end{gathered}
$$

Constraint (19) ensures that following its packet transmissions on channel $\lambda_{c-1}$, the laser at node $i$ has enough time to switch to wavelength $\lambda_{c}$. Constraint (20) is essentially the same as the previous one - it ensures that transmitter $i$ has enough time to tune from channel $\lambda_{C}$ (the last channel) to channel $\lambda_{1}$ to transmit in the next frame. These two constraints correspond to the hardware constraints (5). The no-collision constraints (6) are accounted for in the above description by the constraint $g_{i c} \geq 0 \forall i, c$; by definition of $g_{i c}$, this guarantees that the slots assigned to node $i+1$ on channel $\lambda_{c}$ will be scheduled after the slots assigned to node $i$ in the same channel. 
Note that, finding an optimal schedule within the class (16) for problem $O S T L$ involves solving $N$ ! BW-OSTL problems, one for each possible transmitter sequence, and choosing the schedule of smallest frame size. Furthermore, solving problem $B W$-OSTL is itself a hard task, as it is an integer programming problem with a non-linear objective function, and the size of its state space becomes unmanageable for anything but trivial values of $N$ and $C$.

Recall, however, that we are considering bandwidth limited networks. For such networks, the bandwidth bound (7) dominates, therefore, the lower bound on the schedule length (see (9)) is such that

$$
M^{(l)}=M_{b w}^{(l)}>M_{t}^{(l)}
$$

In other words, there can be no schedule of length less than $M^{(l)}$, as there exists at least one channel $\lambda_{c}$ such that $\sum_{i=1}^{N} a_{i c}=M^{(l)}$. The key observation which we will exploit in the following analysis is that, if a schedule of length $M^{(l)}$ exists, then at least one channel, say, channel $\lambda_{c}$, will never be idle; in terms of the above problem formulation, this schedule will be such that $g_{i c}=0 \forall i$. It will be shown shortly that fixing the values of $g_{i c}$ for one channel makes it possible to solve problem $B W$-OSTL in polynomial time. But first, let us attempt to answer a fundamental question related to the existence of schedules of length $M^{(l)}$ within the class (16).

\subsubsection{A Sufficient Condition for Optimality}

Let $\mathbf{A}$ be the collapsed traffic matrix of a bandwidth limited network, and let $M^{(l)}$ be the lower (bandwidth) bound on any schedule for $\mathrm{A}$. We now define the average slot requirement for a sourcedestination pair as $a=\frac{M^{(l)}}{N}$. Our first observation is that if $a_{i c}=a \forall i, c$, then an optimum length schedule is easy to construct; just let

$$
K_{c}=(c-1)(a+\Delta) \forall c ; g_{i c}=0 \forall i, c ; M=M^{(l)}=N a
$$

and all of (19) - (21) will be satisfied. The question that naturally arises then, is whether we can guarantee a schedule of $M^{(l)}$ slots when we allow non-uniform traffic. The answer is provided by the following lemma.

Lemma 5.1 Let A be a collapsed traffic matrix such that the lower bound in $(9) M^{(l)}=M_{b w}^{(l)}>$ $M_{t}^{(l)}$ (bandwidth limited network). Then, a schedule of length equal to the lower bound, $M^{(l)}$, exists within the class (16) for any transmitter sequence, if the elements of $\mathbf{A}$ satisfy the following condition:

$$
\left|a_{i c}-\frac{M^{(l)}}{N}\right| \leq \epsilon \quad \forall i, c
$$


with $\epsilon$ given by:

$$
\epsilon=\frac{M^{(l)}}{N+1}\left(\frac{1}{C}-\frac{1}{N}-\frac{\Delta}{M^{(l)}}\right)
$$

In proving Lemma 5.1 we will make use of the following result.

Lemma 5.2 If constraints (24) on the elements of $\mathbf{A}$ hold, then for all $\mathcal{P} \subseteq\{1, \ldots, N\}$ with $|\mathcal{P}|=n$, and any two channels $\lambda_{c_{1}}$ and $\lambda_{c_{2}}$ :

$$
\left|\sum_{i \in \mathcal{P}} a_{i, c_{1}}-\sum_{i \in \mathcal{P}} a_{i, c_{2}}\right| \leq N \epsilon
$$

Proof (of Lemma 5.2). Because of (24), for any $n \in\{1, \ldots, N\}$, and any channel $\lambda_{c}$ we get:

$$
n\left(\frac{M^{(l)}}{N}-\epsilon\right) \leq \sum_{i \in \mathcal{P}} a_{i c} \leq M^{(l)}-(N-n)\left(\frac{M^{(l)}}{N}-\epsilon\right)
$$

Given the above, the result in (26) can be easily derived.

We are now ready to prove Lemma 5.1. Note that, although the proof refers to the problem formulation in (18) - (21), it does not depend on the actual transmitter sequence. As a result, it holds for any transmitter sequence, not just the $(1,2, \ldots, N)$ sequence implied in $(18)-(21)$.

Proof (of Lemma 5.1). By our hypothesis, we have that $\sum_{i=1}^{N} a_{i c} \leq M^{(l)} \forall c$. For the proof we consider a worst case scenario, under which the total slot requirement on each channel is equal to the lower bound:

$$
\sum_{i=1}^{N} a_{i c}=M^{(l)} \quad \forall c
$$

A schedule of length $M^{(l)}$ under such a scenario would ensure a schedule of length $M^{(l)}$ for the case when the slot requirement on some channel is less than $M^{(l)}$, as one can simply introduce slots in which this channel is idle.

Since we are trying to achieve a schedule of length $M^{(l)}$, and because of the above worst case assumption, we are seeking a solution to problem $B W$-OSTL such that $g_{i c}=0 \forall i, c$ (refer also to the objective function (18)). We can then rewrite constraints (19) and (20), respectively, as

$$
\begin{gathered}
K_{c}-K_{c-1} \geq\left(\sum_{j=1}^{i-1} a_{j, c-1}-\sum_{j=1}^{i-1} a_{j c}\right)+a_{i, c-1}+\Delta \quad c=2, \ldots, C, i=1, \ldots, N \\
M-K_{C} \geq\left(\sum_{j=1}^{i-1} a_{j C}-\sum_{j=1}^{i-1} a_{j 1}\right)+a_{i C}+\Delta
\end{gathered}
$$


Hence, Lemma 5.2 guarantees that choosing $K_{c}-K_{c-1}=N \epsilon+\frac{M^{(l)}}{N}+\epsilon+\Delta, c=2, \ldots, C$, satisfies constraints (29) and (21). Noting that $K_{1}=0$, we can set:

$$
K_{c}=(c-1)\left((N+1) \epsilon+\frac{M^{(l)}}{N}+\Delta\right) \quad c=1, \ldots, C
$$

Finally, it is easy to check that letting $M=M^{(l)}$ ensures that (30) is also satisfied.

Lemma 5.1 provides an upper bound on the "degree of non-uniformity" of matrix $\mathbf{A}$ in order to guarantee a schedule of length equal to the lower bound. To get a feeling of how restrictive this bound is, let us rewrite expression (25) as

$$
\frac{\epsilon}{M^{(l)} / N}=\frac{N}{N+1}\left(\frac{1}{C}-\frac{1}{N}-\frac{\Delta}{M^{(l)}}\right)
$$

For $N=100, C=10$, and ignoring the term $\frac{\Delta}{M^{(l)}}{ }^{12}$, we get $\frac{\epsilon}{M^{(l)} / N} \approx .089$. Thus, the variation of elements $a_{i c}$ around $\frac{M^{(l)}}{N}$ can be up to $8.9 \%$ to guarantee a schedule of length $M^{(l)}$. Note, however, that the proof of Lemma 5.1 is based on a worst case scenario; in general, we expect such a schedule to exist for significant higher degrees of variation.

As a final observation, $\epsilon$ is greater than zero only when $M^{(l)}>\frac{N C \Delta}{N-C}$. This is consistent with our hypothesis of a bandwidth limited network.

\subsubsection{Scheduling Algorithm}

Lemma 5.1 provides a sufficient condition for the existence of an optimum length schedule, but does not state how to construct one. We now develop a polynomial time scheduling algorithm which, under the conditions of Lemma 5.1, produces schedules of length $M^{(l)}$. In fact, we shall shortly prove that the algorithm is optimal under looser conditions that do not impose any bound on the variation of $a_{i c}$ around $\frac{M^{(l)}}{N}$. The key idea behind the algorithm is to schedule the transmissions on the first channel so that this channel is always busy, except maybe after all nodes have been given a chance to transmit; we expect this strategy to work well when this first channel is the dominant one, that is $\sum_{i=1}^{N} a_{i 1}=M^{(l)}$.

Algorithm Make_Bandwidth_Limited_Schedule (MBLS) is described in detail in Figure 3, and operates as follows. All the gaps in channel $\lambda_{1}$ are initialized to zero; then, during Pass 1, transmissions in channels $\lambda_{2}$ through $\lambda_{C}$ are scheduled at the earliest possible time that satisfies constraints (19). Doing so, however, may introduce large gaps into the channels, resulting in a sub-optimal schedule length (refer to (18)). During the second pass then, the algorithm attempts to compact

\footnotetext{
${ }^{12}$ In general, we expect the frame length to be much greater than $\Delta$.
} 
the gaps within each channel by shifting the slots to the right or left, but only as far as constraints (19) and (20) allow.

That algorithm MBLS is correct follows from the fact that it constructs a schedule which satisfies the constraints (19) - (21), and hence gives an admissible schedule. We now state and prove its optimality properties.

Theorem 5.1 Algorithm MBLS constructs a schedule of minimum length among the schedules that (a) are within the class (16) and the sequence of transmitters is $(1,2, \ldots, N)$, (b) channel $\lambda_{1}$ is a dominant channel, and (c) channel $\lambda_{1}$ is never idle, except, possibly, at the very end of the frame (i.e., $g_{i 1}=0, i=1, \ldots, N-1$ ).

Proof. See Appendix B.

Corollary 5.1 (Optimality of Algorithm MBLS) Let $\lambda_{1}$ be a channel such that $\sum_{i=1}^{N} a_{i 1}=$ $M^{(l)}$, and arbitrarily label the transmitters 1 through $N$. Then, under the conditions of Lemma 5.1, algorithm MBLS constructs an optimum length schedule, i.e., a schedule of length $M^{(l)}$.

Proof. According to Lemma 5.1, there exists a schedule of length $M^{(l)}$ within the class defined by (16), such that the transmitter sequence is $(1,2, \ldots, N)$. Since $\lambda_{1}$ is the dominant channel, any schedule of length $M^{(l)}$ is such that channel $\lambda_{1}$ is never idle. Therefore, because of Theorem 5.1, algorithm MBLS will construct such a schedule.

Regarding the complexity of $M B L S$, it is easy to verify that the algorithm takes time $\mathcal{O}\left(C N^{2}\right)$, regardless of the actual values of the traffic elements $a_{i c}$.

\subsection{Tuning Limited Networks}

The analysis and scheduling algorithm presented in the previous subsection pertain specifically to bandwidth limited networks. We now turn our attention to tuning limited networks. Since our discussion above focused on how transmissions are scheduled within each channel, it is only natural that we now adopt a transmitter's point of view, and concentrate on how its transmissions are scheduled across the various channels ${ }^{13}$.

Let $\mathcal{S}$ be a schedule of length $M$ for a tuning limited network, and let $\left(\lambda_{1}, \ldots, \lambda_{C}\right)$ be the channel sequence for all nodes. For each transmitter we consider the frame that begins with the

\footnotetext{
${ }^{13}$ As the reader will soon notice, the following discussion and results mirror those in the previous subsection. This confirms our intuition that bandwidth limited and tuning limited networks are in a sense dual of each other.
} 
Algorithm Make_Bandwidth_Limited_Schedule (MBLS)

The algorithm assumes that channel $\lambda_{1}$ is dominant. Also, references to channel $\lambda_{c+1}$ when $c=C$ denote the next frame on channel $\lambda_{1}$.

1. begin

2. $\quad$ Set $M=\sum_{i=1}^{N} a_{i 1}$

3. $\quad$ Set $K_{1}$ and all gaps $g_{i 1}$ on $\lambda_{1}$ equal to 0

// Begin Pass 1

4. for $c=2$ to $C$ do

5. $\quad$ for $i=1$ to $N$ do

6. Schedule the $a_{i c}$ slots at the earliest possible time

such that constraint (19) is satisfied between channels $\lambda_{c}$ and $\lambda_{c-1}$

7. $\quad / /$ end of for $c$ loop

// End of Pass 1 - initial values to all $g_{i c}$ have now been determined

8. Let $M^{\prime}$ be the smallest integer satisfying constraint (20)

9. $\quad$ Set $M=\max \left\{M, M^{\prime}\right\}$

// Begin Pass 2

10. for $c=C$ downto $2 \mathrm{do}$

11. for $i=N$ downto 1 do

12. Shift the $a_{i c}$ slots as much right as possible while

maintaining constraint (19) between channels $\lambda_{c}$ and $\lambda_{c+1}$

13. for $j=i+1$ to $N$ do

14. Shift the $a_{j c}$ slots as much left as possible while maintaining constraint (19) between channels $\lambda_{c}$ and $\lambda_{c-1}$

15. // end of for $i$ loop - the final values of gaps for this channel have now been determined

16. Let $M_{c}=\sum_{i=1}^{N}\left(a_{i c}+g_{i c}\right)$

17. $M=\max \left(M, M_{c}\right)$

18. // end of for $c$ loop $-M$ is now the final length of the schedule

19. // end of algorithm

Figure 3: Scheduling algorithm for bandwidth limited networks 


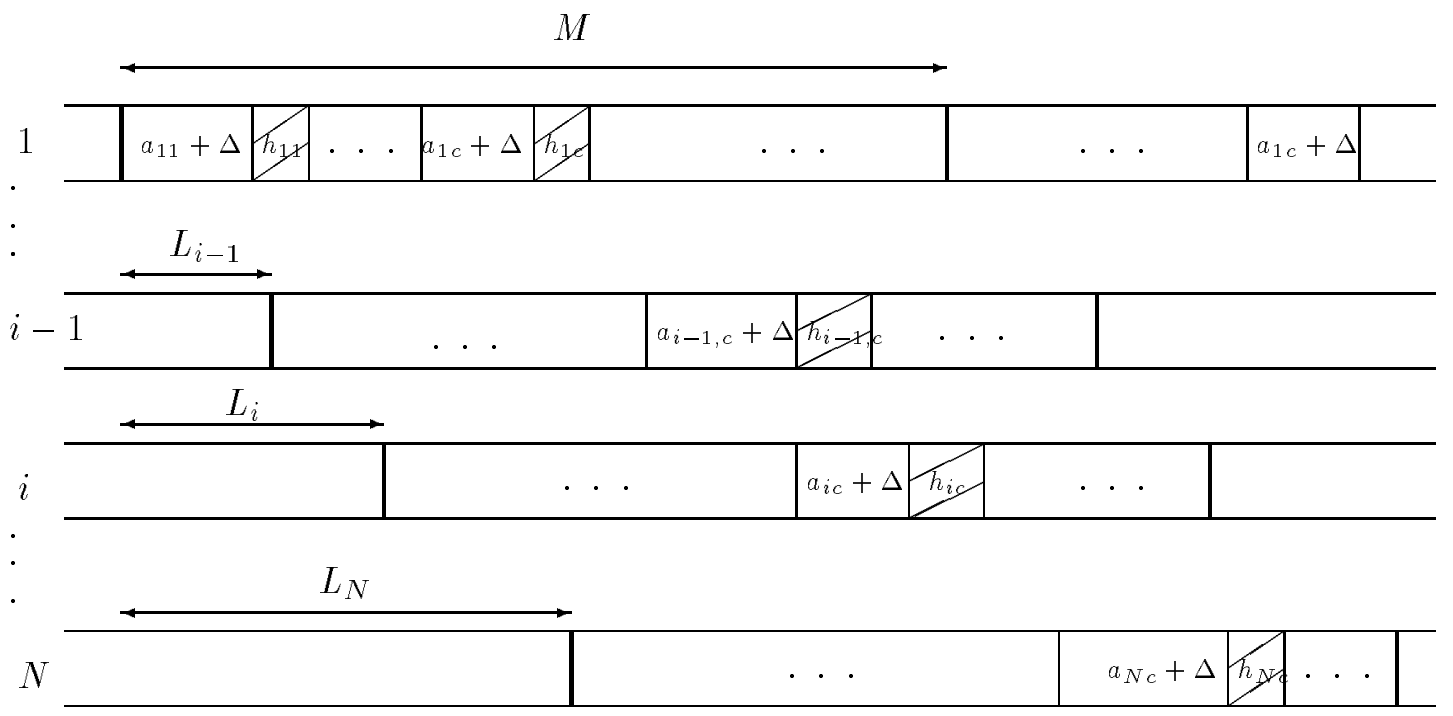

Figure 4: Schedule for a tuning limited network

first slot assigned on channel $\lambda_{1}$. Let the start of the frame for node 1 be our reference point, and let $L_{i}$ denote the distance, in slots, between the start of the frame for node $i$, and the reference point (see Figure 4).

Consider now the transmissions of, say, node $i$, within a frame of $M$ slots. Following its $a_{i 1}$ slots on channel $\lambda_{1}$, and a tuning period of $\Delta$ slots in which it tunes its laser to $\lambda_{2}$, node $i$ is ready to transmit on that channel. However, node $i-1$ may still be transmitting on $\lambda_{2}$, in which case node $i$ will remain idle for several slots before it starts transmitting on $\lambda_{2}$. In general, we let $h_{i c}$ denote the number of slots transmitter $i$ remains idle between the time it has tuned its laser to channel $\lambda_{c+1}$ and the start of its transmissions on the same channel.

Based on these observations, the problem of finding an optimum length schedule such that (a) the schedule is within the class (17) ${ }^{14}$, and (b) the channel sequence is $\left(\lambda_{1}, \ldots, \lambda_{C}\right)$ can be formulated as an integer programming problem, referred to as tuning limited OSTL (T-OSTL):

$$
T-O S T L: \quad \min _{h_{i c}, L_{i}} M=\max _{i}\left\{\sum_{c=1}^{C}\left(a_{i c}+\Delta+h_{i c}\right)\right\}
$$

subject to:

$$
L_{i}+\sum_{l=1}^{c-1}\left(a_{i l}+\Delta+h_{i l}\right) \geq L_{i-1}+\sum_{l=1}^{c-1}\left(a_{i-1, l}+\Delta+h_{i-1, l}\right)+a_{i-1, c} \quad i=2, \ldots, N, c=1, \ldots, C
$$

\footnotetext{
${ }^{14}$ Recall that classes (17) and (16) are equivalent.
} 


$$
\begin{gathered}
M+\sum_{l=1}^{c-1}\left(a_{1 l}+\Delta+h_{1 l}\right) \geq L_{N}+\sum_{l=1}^{c-1}\left(a_{N l}+\Delta+h_{N l}\right)+a_{N c} \quad i=1, \ldots, N \\
h_{i c}, L_{i}, M \text { : integers; } h_{i c} \geq 0 \forall i, c ; L_{1}=0 ; L_{i}>L_{i-1} i=2, \ldots, N ; M>L_{N}
\end{gathered}
$$

Constraints (34) and (35) are essentially the no-collision constraints (6); they ensure that transmissions by some node $i$ on some channel $\lambda_{c}$ start after the end of transmissions by node $i-1$ on the same channel. The hardware constraints (5) correspond to constraints $h_{i c} \geq 0 \forall i, c$.

Note that the above formulation is very similar to the one in (18) - (21), and that problem $T$-OSTL is equally as hard to solve as $B W$-OSTL. However, in the case we are considering, the tuning bound dominates, thus

$$
M^{(l)}=M_{t}^{(l)}>M_{b w}^{(l)}
$$

In other words, there exists a transmitter $i$ such that $\sum_{c=1}^{C}\left(a_{i c}+\Delta\right)=M^{(l)}$. Using this observation we were able to prove optimality properties and derive a scheduling algorithm for tuning limited networks. In the following, however, the various proofs are omitted, as they are very similar to the corresponding proofs for the bandwidth limited case presented earlier.

\subsubsection{A Sufficient Condition for Optimality}

For tuning limited networks we define the average slot requirement (which now includes both transmission and tuning slots) as $a^{\prime}=\frac{M^{(l)}}{C}$. Then, the following lemma, analogous to Lemma 5.1, provides a sufficient condition for the existence of a schedule of length $M^{(l)}$.

Lemma 5.3 Let A be a collapsed traffic matrix such that the lower bound in $(9) M^{(l)}=M_{t}^{(l)}>$ $M_{b w}^{(l)}$ (tuning limited network). Then, a schedule of length equal to the lower bound, $M^{(l)}$, exists within the class (17) for any channel sequence, if the elements of A satisfy the following condition:

$$
\left|\left(a_{i c}+\Delta\right)-\frac{M^{(l)}}{C}\right| \leq \epsilon^{\prime} \quad \forall i, c
$$

with $\epsilon^{\prime}$ given by:

$$
\epsilon^{\prime}=\frac{M^{(l)}}{C+1}\left(\frac{\Delta}{M^{(l)}}+\frac{1}{N}-\frac{1}{C}\right)
$$

The proof of Lemma 5.3, uses the following result, similar to Lemma 5.4. Both proofs are omitted. Observe also that $\epsilon^{\prime}$ is greater than zero only when $M^{(l)}<\frac{N C \Delta}{N-C}$. 
Lemma 5.4 If constraints (38) on the elements of $\mathbf{A}$ hold, then for all $\mathcal{Q} \subseteq\{1, \ldots, C\}$ with $|\mathcal{Q}|=k$, and any two transmitters, $i$ and $j$ :

$$
\left|\sum_{c \in \mathcal{Q}}\left(a_{i c}+\Delta\right)-\sum_{c \in \mathcal{Q}}\left(a_{j c}+\Delta\right)\right| \leq C \epsilon^{\prime}
$$

\subsubsection{Scheduling Algorithm}

As in the case of bandwidth limited schedules, we have developed a scheduling algorithm for tuning limited networks which is optimal under the conditions of Lemma 5.3. This algorithm, which we will call Make_Tuning_Limited_Schedule (MTLS), is very similar to MBLS, and is omitted. The key idea is to schedule the slots of a certain node so that its transmitter is never idle, except possibly at the end of a frame. The optimality properties of the MTLS algorithm are now stated without proof.

Theorem 5.2 Algorithm MTLS constructs a schedule of minimum length among the schedules that (a) are within the class (17) and the sequence of channels is $\left(\lambda_{1}, \ldots, \lambda_{C}\right),(b)$ transmitter 1 is a dominant transmitter, and (c) transmitter 1 is never idle, except, possibly, at the very end of the frame (i.e., $h_{1 c}=0, c=1, \ldots, C-1$ ).

Corollary 5.2 (Optimality of Algorithm MTLS) Let transmitter 1 be such that $\sum_{c=1}^{C}\left(a_{1 c}+\right.$ $\Delta)=M^{(l)}$, and arbitrarily label the channels $\lambda_{1}$ through $\lambda_{C}$. Then, under the conditions of Lemma 5.3, algorithm MBLS constructs an optimum length schedule, i.e., a schedule of length $M^{(l)}$.

\subsection{Tuning and Bandwidth Balanced Networks}

The last two subsections considered the operation of the network in two distinct regions, the bandwidth and tuning limited regions, respectively. We now study the effect of operating the network at the boundary of the two regions, namely, when the tuning and bandwidth bounds are equal:

$$
M^{(l)}=M_{t}^{(l)}=M_{b w}^{(l)}
$$

In particular, we show that if (41) holds, even arbitrarily small non-uniformities in the traffic pattern may result in every admissible schedule having length greater than the lower bound $M^{(l)}$. Our claim is stated in the following theorem; note that neither the theorem nor its proof refer to the class of schedules defined by (16) and (17), therefore, this result holds for arbitrary schedules, not only the ones implied by (16) or (17). 
Theorem 5.3 Let A be a collapsed traffic matrix such that the bandwidth and tuning bounds are equal, and such that each transmitter and each channel are tight (i.e., the slot requirement on each channel is equal to the lower bound, and the slot-plus-tuning requirement of each transmitter is also equal to the lower bound). Then, the optimal schedule has length strictly greater than the lower bound, even for any arbitrarily small non-uniformity among the elements of matrix A.

Proof. We will construct one such instance of the $O S T L$ problem and prove that a schedule for this instance will necessarily have length strictly greater than the lower bound; this instance can be easily generalized by scaling the values of the various parameters.

Consider a system of $N=4$ nodes, $C=3$ channels, and $\Delta=1$. Let the matrix A be such that

$$
a_{11}=a_{22}=3-\epsilon, \quad a_{21}=a_{12}=3+\epsilon, \quad a_{i c}=3 \text { forall other } i, c
$$

for some arbitrary $\epsilon<1$. It is easy to verify that $M_{t}^{(l)}=M_{b w}^{(l)}=12$, and that all channels and transmitters are tight.

Suppose now that a schedule of length 12 exists. Then neither any channel nor any transmitter can be in the idle at any time instant in such a schedule. Consider any frame of the schedule starting at time $t$ in which node 1 transmits on channel $\lambda_{3}$. In other words, node 1 transmits on channel $\lambda_{3}$ from time $t$ to time $t+3$. Since transmitter 1 is tight and the frames repeat over time, node 1 must, at time $t+4$, start transmitting on some other channel. Without loss of generality, let $\lambda_{1}$ be this other channel, in which case node 1 will transmit on channel $\lambda_{1}$ from time $t+4$ till time $t+7-\epsilon$, and on channel $\lambda_{2}$ from time $t+8-\epsilon$ till time $t+11$.

Let $t$ be integral (if it is not, we can appropriately shift the start time of the schedule to make $t$ an integer). Note that all transmissions on channel $\lambda_{3}$ begin and end at integral time values. Further, since nodes 3 and 4 have integral slot requirements on each channel, their start and end times on all channels have to be integral; only the transmissions of nodes 1 and 2 on channels $\lambda_{1}$ and $\lambda_{2}$ will be skewed $\epsilon$ away from integral values. So when node 1 finishes transmitting on $\lambda_{1}$ at time $t+7-\epsilon$, node 2 is the only candidate for immediate transmission on $\lambda_{1}$ (which, recall, must never be idle). But if node 2 were to start transmitting at time $t+7-\epsilon$ on $\lambda_{1}$, it must have finished transmission at time $t+6-\epsilon$ on some other channel (again, recall that no node must ever be idle), and that channel has to be $\lambda_{2}$, since $t+6-\epsilon$ is non-integral. Therefore, node 2 must have started transmission on $\lambda_{2}$ at time $t+3$, which implies that it must have transmitted on channel $\lambda_{3}$ from time $t-1$ to time $t+2$. But this overlaps with the transmission of node 1 on the same channel, contradicting our hypothesis that an admissible schedule of length equal to the lower bound $(=12)$ exists. 


\section{Optimization Heuristics}

In the previous section we presented scheduling algorithms which construct schedules of length equal to the lower bound as long as traffic parameters $a_{i c}$ satisfy certain conditions. However, we have seen that $O S T L$ is $\mathcal{N} \mathcal{P}$-complete, meaning that an algorithm that efficiently solves any arbitrary instance of $O S T L$ (i.e., one in which $a_{i c}$ may not satisfy the optimality conditions) may not exist. Hence, the most we can hope for is to be able to devise a heuristic that can be expected to perform well for general traffic matrices. We now develop a scheduling heuristic for bandwidth limited networks; using a very similar reasoning, it is relatively straightforward to determine a heuristic for tuning limited networks.

Recall that for bandwidth limited networks, finding a schedule within the class (16) that solves the $O S T L$ problem involves solving $N ! B W$-OSTL problems (see (18) - (21)), one for each possible transmitter sequence; obviously, no polynomial-time heuristic can consider that many sequences. On the other hand, we have no efficient algorithm for solving the most general version of $B W$ $O S T L$, but we have developed $M B L S$, a polynomial time algorithm that solves $B W$-OSTL for a given transmitter sequence under the additional constraint that any idling of the first channel occurs after all nodes have transmitted on that channel (refer also to Theorem 5.1). With these considerations in mind, our approach to obtaining near-optimal schedules for $O S T L$ is based on making two compromises:

- Suppose that an optimal transceiver sequence for a network of $n$ nodes has been determined, and that a new node is added to the network (that is, a new row is added to the collapsed traffic matrix A, while all other elements remain as before). Instead of checking all possible $(n+1)$ ! transmitter sequences, our first approximation is to assume that, in the optimal sequence for the $(n+1)$-node network, the relative positions of nodes 1 through $n$ are the same as in the sequence for the $n$-node network; thus, we only need to determine where in the latter sequence node $n+1$ has to be inserted (i.e., before the first node, between the first and second nodes, etc.). This can be accomplished by solving $n+1 B W$-OSTL problems on a $(n+1)$-node network, one for each possible placement of node $n+1$ within the initial sequence of $n$ nodes.

- Our second compromise has to do with the fact that we have no efficient algorithm for $B W$ $O S T L$. Thus, we let $\lambda_{1}$ be the dominant channel, and use algorithm MBLS to solve the version of $B W$-OSTL which requires that $\lambda_{1}$ is never idle except at the end of the frame. From Theorem 5.1, we know that if a schedule of length equal to the lower bound exists for the given transmitter sequence, $M B L S$ will find such a schedule. But if the optimal schedule 


\section{Bandwidth Limited Scheduling Heuristic (BLSH)}

1. Relabel the channels such that:

$$
M^{(l)}=\sum_{i=1}^{N} a_{i 1} \geq \sum_{i=1}^{N} a_{i 2} \geq \ldots \geq \sum_{i=1}^{N} a_{i C}
$$

Arbitrarily label the transmitters as $1, \ldots, N$, and let $s^{(1)}=(1)$. Repeat Step 2 for $i=$ $2, \ldots, N$.

2. Let $s^{(i-1)}=\left(\pi_{1}, \ldots, \pi_{i-1}\right)$ be the permutation produced by the previous iteration on a network with only the first $i-1$ transmitters of the original network. Consider transmitter $i$. Run algorithm MBLS on each of the $i$ permutations

$$
\left(i, \pi_{1}, \ldots, \pi_{i-1}\right),\left(\pi_{1}, i, \pi_{2}, \ldots, \pi_{i-1}\right), \ldots,\left(\pi_{1}, \ldots, \pi_{j}, i, \pi_{j+1}, \ldots, \pi_{i-1}\right), \ldots,\left(\pi_{1}, \ldots, \pi_{i-1}, i\right)
$$

Let $s^{(i)}$ be the permutation that results in the least length schedule.

Figure 5: Scheduling Heuristic

has length greater than the lower bound, MBLS may fail to produce an optimal solution as the idling in the first channel may be anywhere within the frame, not necessarily at the end. Numerical results to be presented, however, do suggest that overall the performance of $M B L S$ is very close to being optimal.

For bandwidth limited networks, our heuristic is described in Figure 5. About the complexity of the heuristic, note that Step 2 will dominate. During the $i$-th iteration of Step 2, algorithm $M B L S$ is called $i$ times on a network of $i$ nodes. Since the complexity of $M B L S$ on a network of $i$ nodes is $\mathcal{O}\left(C i^{2}\right)$, the overall complexity of the heuristic is $\mathcal{O}\left(C N^{4}\right)$.

\section{$7 \quad$ Numerical Results}

We now consider four different algorithms for the OSTL problem and compare their performance. The four algorithms are:

1. algorithm $M B L S$, described in Figure 3; the algorithm is applied after the channels have been labeled $\lambda_{1}$ through $\lambda_{C}$ in decreasing order of $\sum_{i=1}^{N} a_{i c}$, and the transmitters have been labeled 
1 through $N$ in decreasing order of $\sum_{c=1}^{C} a_{i c}$;

2. algorithm MTLS, with the same labeling of both channels and transmitters; the algorithm has not been described here, but is very similar to $M B L S$, only targeted to tuning limited networks;

3. scheduling heuristic BLSH, described in Figure 5;

4. scheduling heuristic TLSH for tuning limited networks; again, this heuristic has not been described, but is very similar to $B L S H$.

We have generated random instances of the $O S T L$ problem, i.e., random matrices $\mathbf{A}$ for various values of $N, C$, and $\Delta$, and fed them as input to the algorithms. Given $\mathbf{A}$, the lower bound $M^{(l)}$ on the schedule length can be obtained from (9), (8) and (7). Let $M$ be the actual length of a schedule for A produced by some scheduling algorithm. Then the quantity

$$
\frac{M}{M^{(l)}} 100 \%
$$

represents how far the length $M$ of the schedule produced by the algorithm is from the lower bound. All figures in this section plot the quantity in (45) against the number of nodes, $N$, for the five algorithms described above. Each point plotted represents the average of twenty different matrices A for the stated values of $N, C$, and $\Delta$.

For the results shown in Figures $6-17$, the elements of each matrix A were chosen, with equal probability, among the integers 1 through 20 (this is the uniform $(1,20)$ distribution). We show four sets of three figures each, corresponding to the four values of the number of channels $C=5,10,15,20$. Within each set with the same value of $C$ we use three different values for $\Delta$, namely $\Delta=1,4,16$. The number $N$ of nodes within each figure takes values from $C$ to 80 . Note that, for data rates of 1 Gigabits per second, and packet sizes equal to the ATM cell size (53 bytes), the packet transmission time (slot length) is $424 n s$. Hence the three values of $\Delta$ considered here correspond to transceiver tuning times of $424 \mathrm{~ns}, 1.7 \mu \mathrm{s}$, and $6.8 \mu \mathrm{s}$, respectively; the last two values are representative of the current state of the art in optical transceiver technology [3].

Let us now concentrate on the relative performance of the four algorithms. Our first observation is that the two heuristics, BLSH and TLSH, always perform as good as, or better than the corresponding algorithms, MBLS and MTLS, respectively. This is in fact expected. As explained above, we applied algorithms $M B L S$ and $M T L S$ to a single transmitter and channel sequence; since the random traffic matrices do not necessarily satisfy the optimality conditions of Lemmas 5.1 and 5.3, these algorithms may fail to produce an optimal schedule. Heuristic BLSH (respectively, TLSH) 
on the other hand, calls $M B L S$ (respectively, MTLS) on several transmitter (respectively, channel) sequences, and is more likely to construct schedules of length close to the lower bound.

The results also confirm our intuition regarding the two regions of network operation, and justify the need for algorithms specially designed for each region. Let us, for the moment, refer to Figure 11 which shows results for $C=10, \Delta=16$. As we can see, algorithms $M B L S$ and $B L S H$ outperform their counterparts, MTLS and TLSH, respectively, when $N>25$, while the opposite is true for $N<25$. Indeed, for these values of $C$ and $\Delta$, and the way the traffic matrices are constructed, a network is in the bandwidth limited region if it has more than 25 nodes, and in the tuning limited region, otherwise (see 15). It should come as no surprise, then, that algorithms $M B L S$ and $B L S H$ (respectively, MTLS and $T L S H$ ), designed for bandwidth (respectively, tuning) limited networks, perform very close to the optimal within their intended region of operation, and sub-optimally within the other. Very similar observations can be made for all other figures presented here.

The figures can also explain how the point at which the network becomes tuning or bandwidth limited depends on the system parameters $N, C$, and $\Delta$ (the traffic parameters are the same for all figures, so they do not play a role at this point; we will consider their effect shortly). Consider Figures $9-11$, corresponding to the same value of $C=10$. As the value of $\Delta$ increases, a larger number of nodes $N$ is needed if expression (15) is to be satisfied ${ }^{15}$. This is indeed reflected in the above figures, as the point at which algorithms MBLS and BLSH outperform MTLS and TLSH, respectively, moves to the right (i.e., towards greater values of $N$ ) as $\Delta$ increases from 1 to 16 . Similarly, if we concentrate on how the number $C$ of channels alone affects the region of operation, we can see from, say, Figures $7,10,13$, and 16 , all of which show results with $\Delta=4$, that for larger $C$ it takes more nodes to keep the network in the bandwidth limited region; this is in accordance to $(15)$, as expected.

Let us now consider the performance of $M B L S$ and $B L S H$ for bandwidth limited networks; very similar conclusions can be drawn regarding the performance of MTLS and TLSH in the tuning limited region. From the various figures we observe that, in general, the length of schedules produced by $M B L S$ and $B L S H$ are very close to the lower bound, and that, for networks well within the bandwidth limited region (i.e., for sufficiently large $N$ ), $B L S H$, and sometimes $M B L S$, construct schedules of length equal to the lower bound. This is a very important result, as it establishes that the lower bound accurately characterizes the scheduling efficiency in this type of environment. Since the lower bound is independent of the tuning latency in this region, this result also implies that it is possible to appropriately dimension the network to eliminate the effects of even large values of

\footnotetext{
${ }^{15}$ Note that the total traffic demand $D$ in (15) can be expressed as $N C \alpha$, where $\alpha$ is the average transmitter-channel slot requirement. For the uniform $(1,20)$ distribution we consider here, $\alpha=10.5$.
} 
tuning latency.

Another important observation is that, although the four algorithms perform very close to the lower bound within their respective regions of operation, they deviate from it for values of $N$ in the boundary of the tuning and bandwidth limited regions (although they are never more than $30 \%$ away from the optimal, and in many cases they are as close as 15\%). This can be explained by noting that, for those values of $N$, the tuning and bandwidth bounds are close to each other. When there are several channels and nodes with similar slot requirements, the scheduling algorithms have less flexibility in placing the various slots to obtain schedules of length close to the lower bound. A similar behavior was observed in [4] for SRA, a scheduling algorithm that operates under a totally different strategy. Combining these results with theorem 5.3 suggests that the behavior of our algorithms in the boundary between the tuning and bandwidth limited regions is not due to inherent inefficiency, but is rather due to the fact that the optimal schedules in this region have length greater than the lower bound.

We now study the effect of the traffic demands on the operation of the network. In Figure 18 we plot the performance of the four algorithms for $C=10$ channels and $\Delta=16$ tuning slots. In this case, however, the elements of matrix A were selected from the uniform $(1,40)$ distribution. Comparing to Figure 11 which plots results for the same values of $C$ and $\Delta$, but with the traffic parameters $a_{i c}$ selected from the uniform $(1,20)$ distribution, we see that the point at which the network becomes bandwidth limited has moved to the left (i.e., a smaller number of nodes); this is in accordance to (15). We also observe that the behavior of the four algorithms is very similar to the one observed before. Finally, in Figure 19 we plot results for $C=10$ and $\Delta=16$, but now elements $a_{i c}$ have been selected according to a bimodal distribution as follows: with probability $\frac{1}{2}$ an element is chosen from the uniform $(1,7)$ distribution, and with probability $\frac{1}{2}$ from the uniform $(14,20)$ distribution (the mean, however, is the same as the uniform(1,20) distribution in Figure 11). As we can see the plots of Figure 19 are very similar to the ones in Figure 11, especially within the bandwidth and tuning limited regions. In general, we have noticed that the quality of the schedules produced by the four algorithms is not significantly affected by the actual distribution of the traffic demands.

Based on the results presented here, we conclude that BLSH and TLSH achieve the best performance within the bandwidth and tuning limited regions, respectively. Algorithms $M B L S$ and MTLS can achieve almost similar performance, but they are more efficient in terms of their running time requirements. The best algorithm for a given system will then depend not only on the region of operation, but also on the desired tradeoff between quality of the final schedule and speed. 


\section{Concluding Remarks}

We have considered the problem of designing TDM schedules to accommodate arbitrary traffic demands in a broadcast optical network. Our objective was to investigate the effects of transceiver tuning latency on the length of the schedule, which is a measure of both delay and throughput. Based on the insight provided by an appropriate new formulation of the scheduling problem, we presented algorithms which construct schedules of length very close to, or equal to the lower bound. We also established that, as long as the network operates within the bandwidth limited region (as determined by system parameters such as the number of nodes, the number of wavelengths, and the number of tuning slots), even large (relative to the packet transmission time) values of the tuning latency have no effect on the length of the schedule. The main conclusion of our work is that through careful design, it is possible to realize single-hop WDM networks operating at very high data rates, using currently available optical tunable devices. Our current research focuses on the design of distributed protocols that will reliably provide the global information required by the algorithms presented here. 


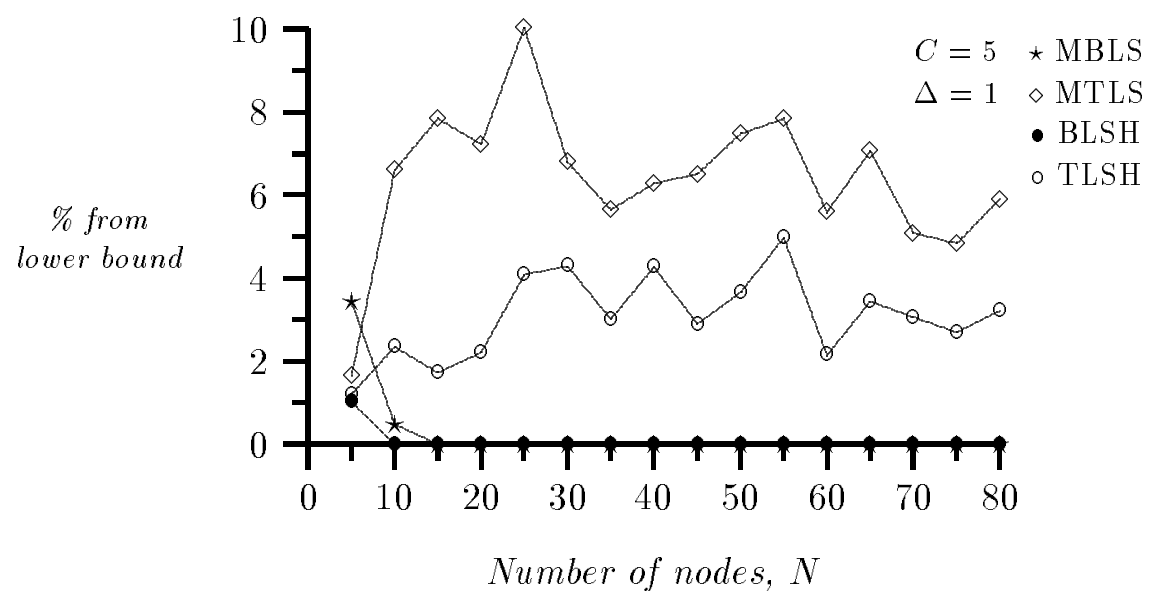

Figure 6: Algorithm comparison for $C=5$ channels and $\Delta=1$ tuning slots

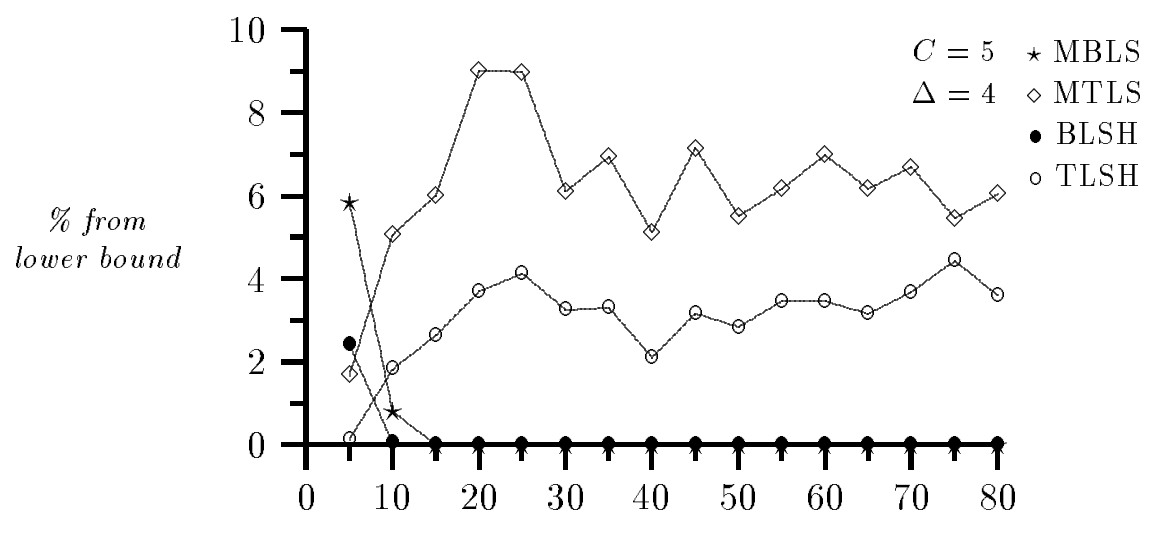

Number of nodes, $N$

Figure 7: Algorithm comparison for $C=5$ channels and $\Delta=4$ tuning slots

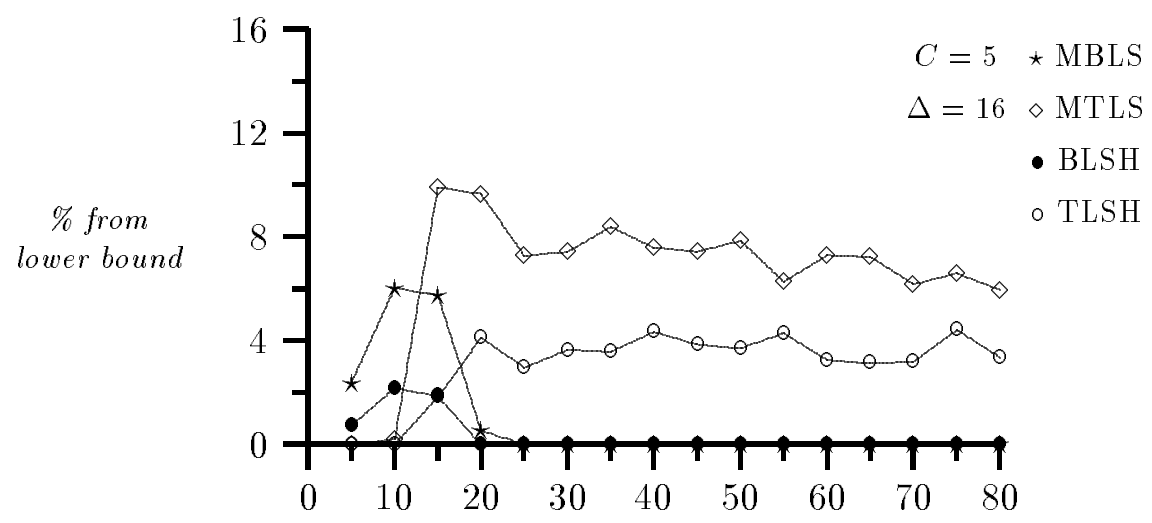

Number of nodes, $N$

Figure 8: Algorithm comparison for $C=5$ channels and $\Delta=16$ tuning slots 


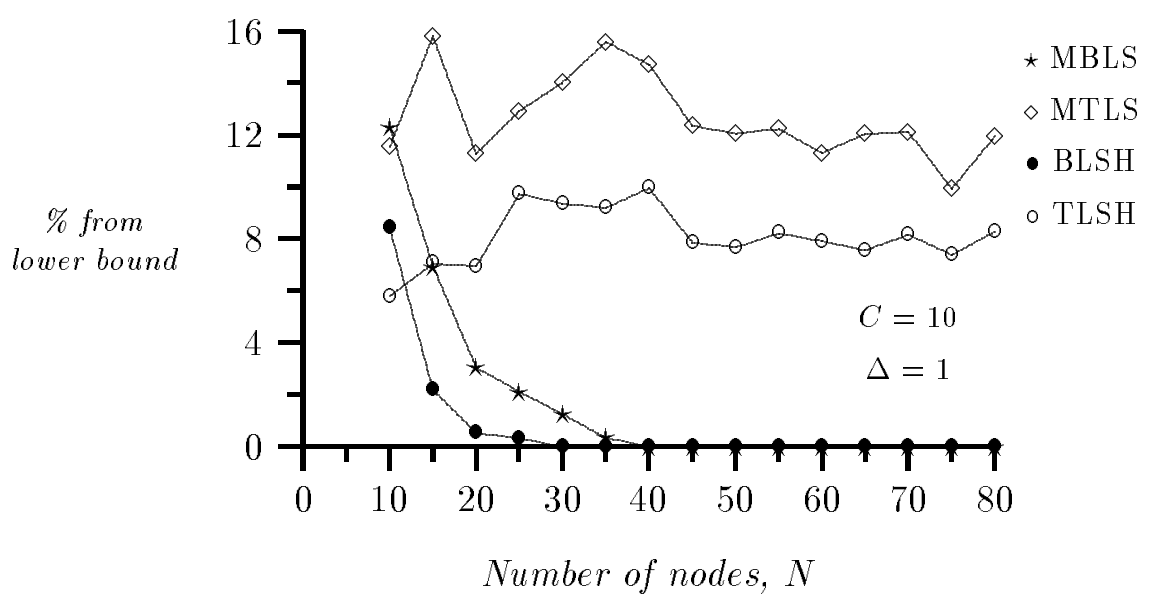

Figure 9: Algorithm comparison for $C=10$ channels and $\Delta=1$ tuning slots

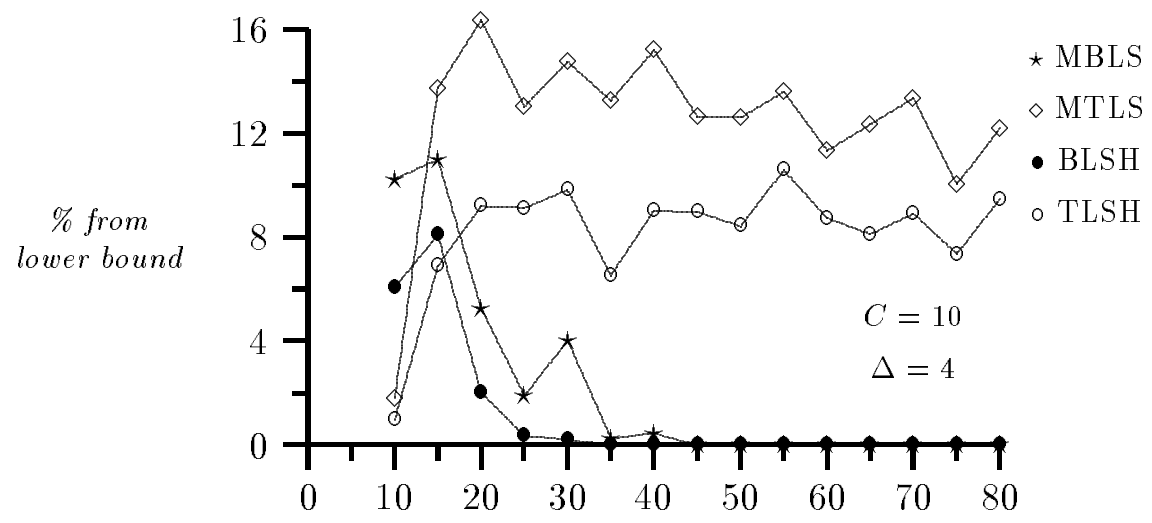

Number of nodes, $N$

Figure 10: Algorithm comparison for $C=10$ channels and $\Delta=4$ tuning slots

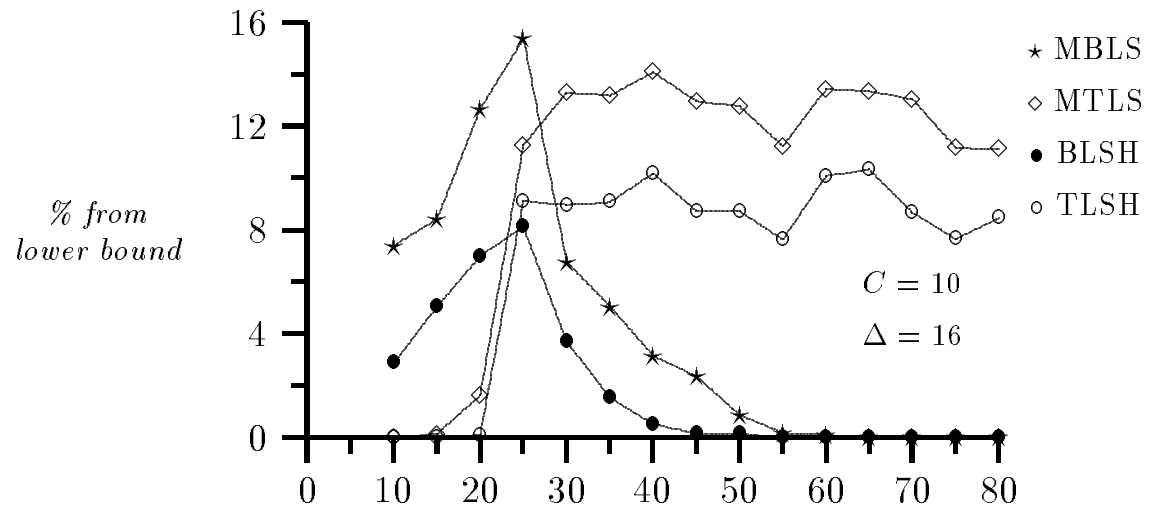

Number of nodes, $N$

Figure 11: Algorithm comparison for $C=10$ channels and $\Delta=16$ tuning slots 


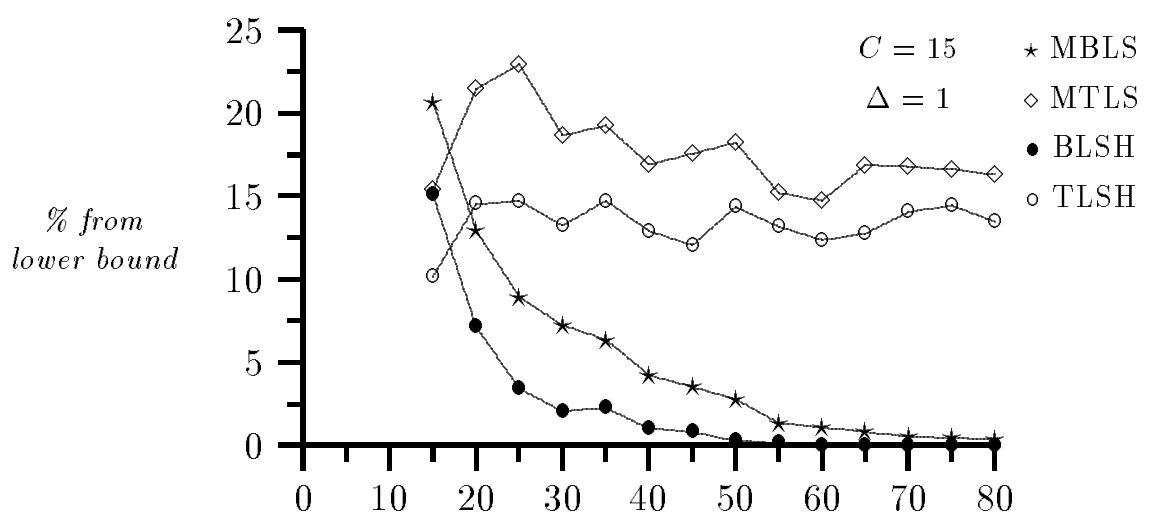

Number of nodes, $N$

Figure 12: Algorithm comparison for $C=15$ channels and $\Delta=1$ tuning slots

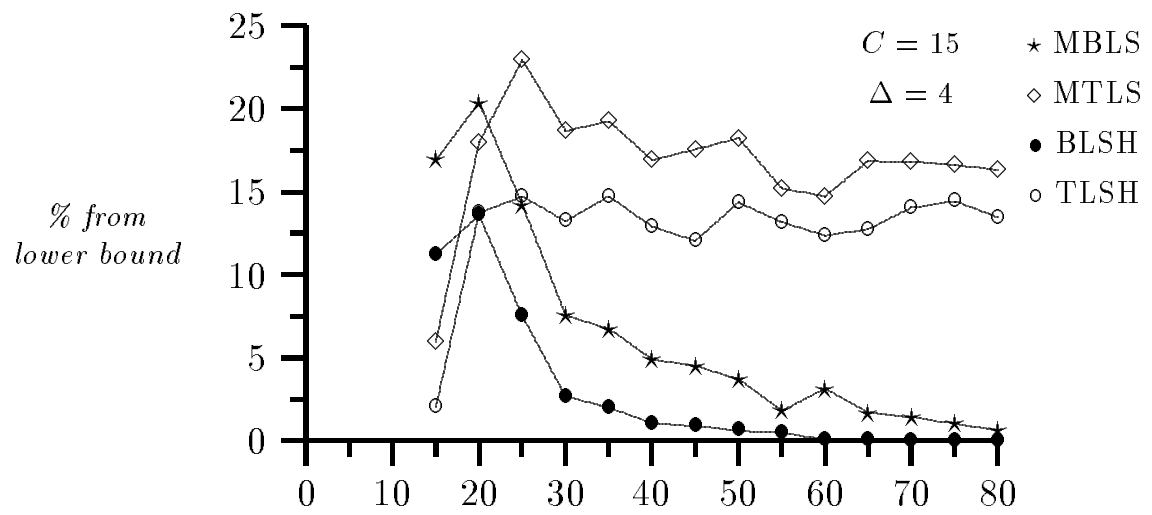

Number of nodes, $N$

Figure 13: Algorithm comparison for $C=15$ channels and $\Delta=4$ tuning slots

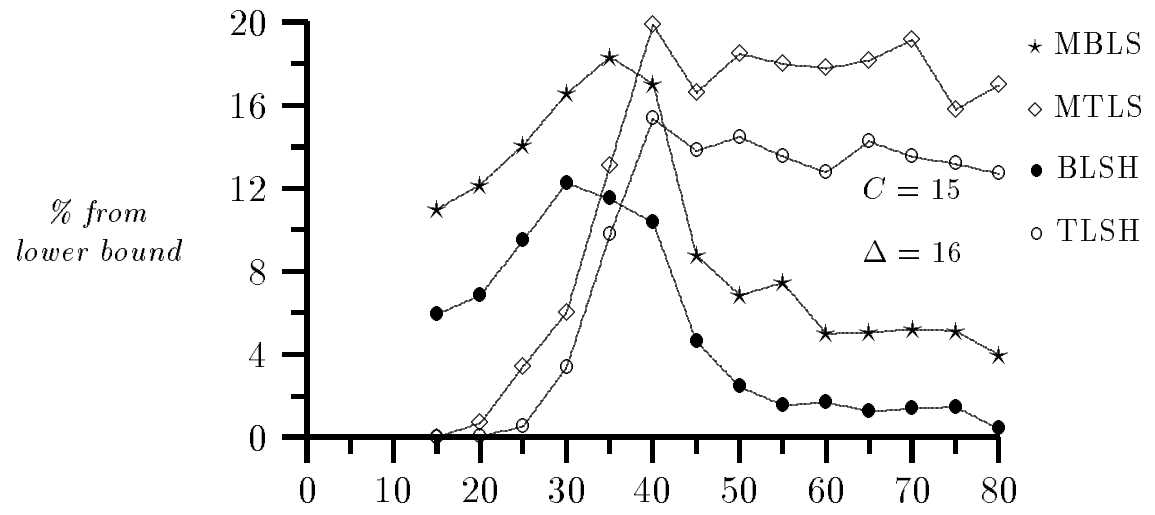

Number of nodes, $N$

Figure 14: Algorithm comparison for $C=15$ channels and $\Delta=16$ tuning slots 


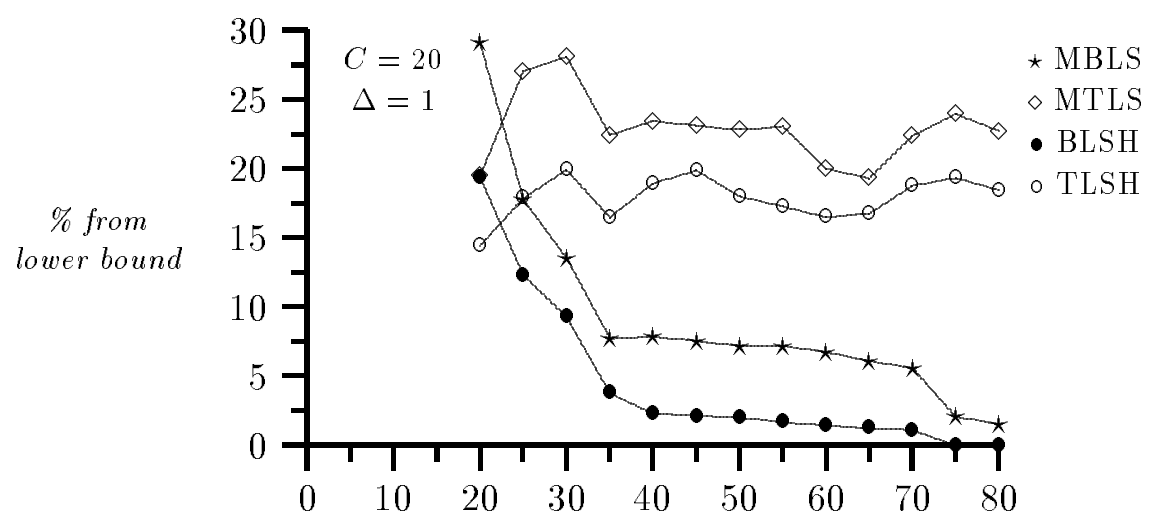

Number of nodes, $N$

Figure 15: Algorithm comparison for $C=20$ channels and $\Delta=1$ tuning slots

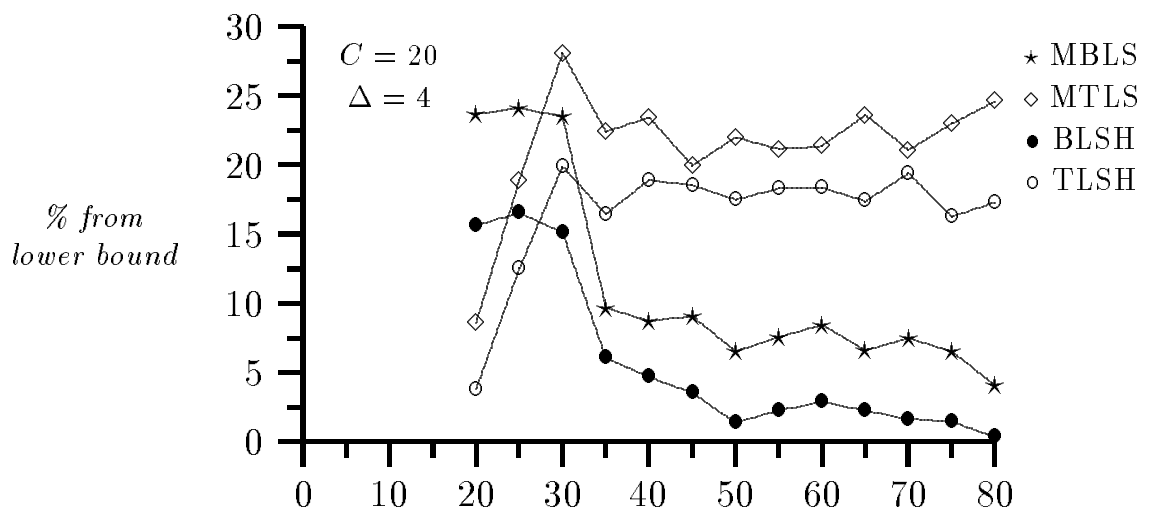

Number of nodes, $N$

Figure 16: Algorithm comparison for $C=20$ channels and $\Delta=4$ tuning slots

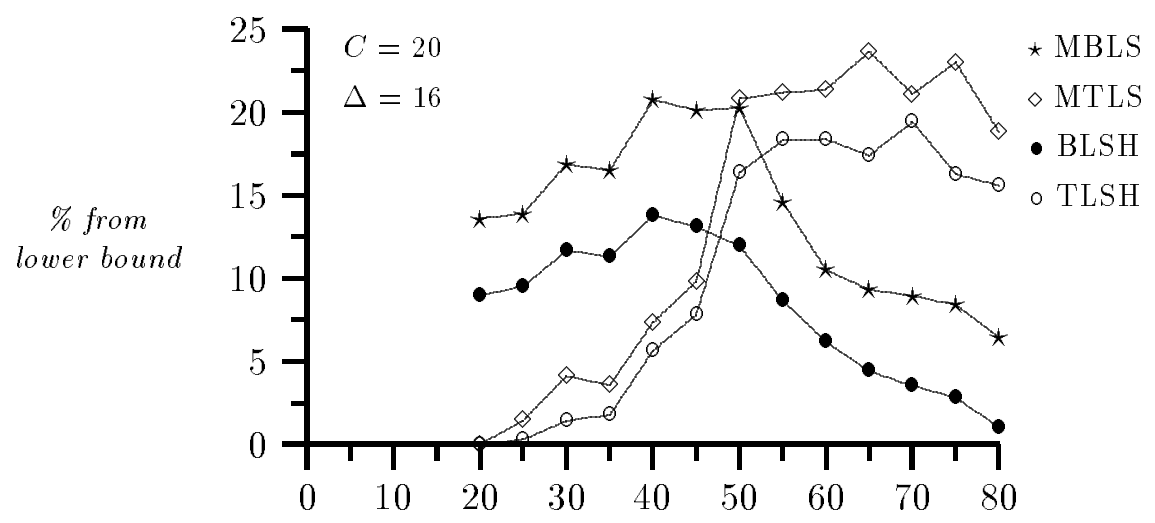

Number of nodes, $N$

Figure 17: Algorithm comparison for $C=20$ channels and $\Delta=16$ tuning slots 


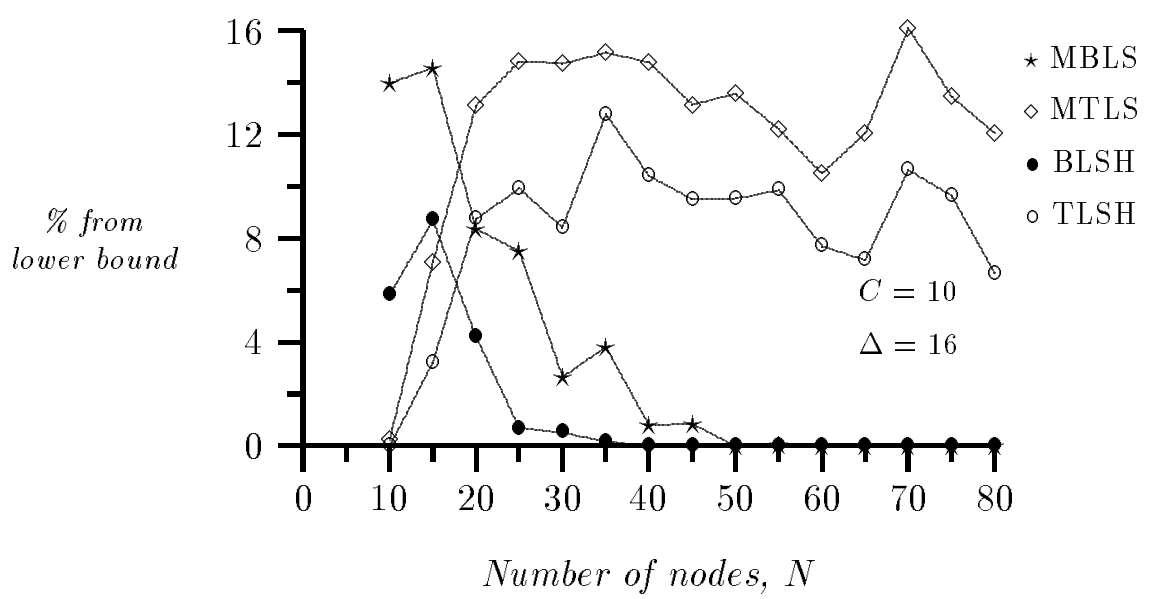

Figure 18: Algorithm comparison for $C=10$ channels and $\Delta=16$ tuning slots (uniform $(1,40$ ) distribution)

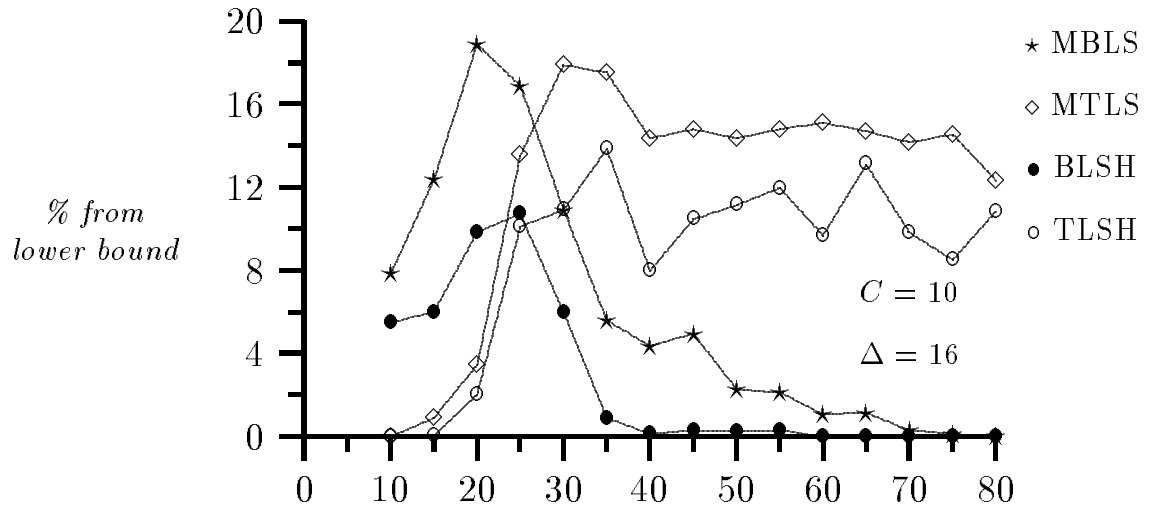

Number of nodes, $N$

Figure 19: Algorithm comparison for $C=10$ channels and $\Delta=16$ tuning slots (bimodal distribution) 


\section{References}

[1] B. Mukherjee. WDM-Based local lightwave networks Part I: Single-hop systems. IEEE Network Magazine, pages 12-27, May 1992.

[2] P. S. Henry. High-capacity lightwave local area networks. IEEE Communication Magazine, pages 20-26, October 1989.

[3] P. E. Green. Fiber Optic Networks. Prentice-Hall, Englewood Cliffs, New Jersey, 1993.

[4] M. Azizoglu, R. A. Barry, and A. Mokhtar. The effects of tuning time in banwidth-limited optical broadcast networks. In Proceedings of INFOCOM'95, pages 138-145. IEEE, April 1995.

[5] G. N. Rouskas and M. H. Ammar. Analysis and optimization of transmission schedules for single-hop WDM networks. IEEE/ACM Transactions on Networking, 3(2):211-221, April 1995.

[6] G. N. Rouskas and M. H. Ammar. Minimizing delay and packet loss in single-hop lightwave WDM networks using TDM schedules. In Proceedings of ICC '95, June 1995.

[7] K. Bogineni, K. M. Sivalingam, and P. W. Dowd. Low-complexity multiple access protocols for wavelength-division multiplexed photonic networks. IEEE Journal on Selected Areas in Communications, 11(4):590-604, May 1993.

[8] Mon-Song Chen, N. R. Dono, and R. Ramaswami. A media-access protocol for packet-switched wavelength division multiaccess metropolitan area networks. IEEE Journal on Selected Areas in Communications, 8(6):1048-1057, August 1990.

[9] R. Chipalkatti, Z. Zhang, and A. S. Acampora. Protocols for optical star-coupler network using WDM: Performance and complexity study. IEEE Journal on Selected Areas in Communications, 11(4):579-589, May 1993.

[10] P. A. Humblet, R. Ramaswami, and K. N. Sivarajan. An efficient communication protocol for high-speed packet-switched multichannel networks. IEEE Journal on Selected Areas in Communications, 11(4):568-578, May 1993.

[11] M. Kovacevic, M. Gerla, and J. Bannister. Time and wavelength division multiaccess with acoustooptic tunable filters. Journal of Fiber and Integrated Optics, 12:113-132, August 1993. 
[12] A. Ganz and Y. Gao. Time-wavelength assignment algorithms for high performance WDM star based networks. IEEE Transactions on Communications, 42(4):1827-1836, April 1994.

[13] S. Tridandapani, J. S. Meditch, and A. K. Somani. The MaTPi protocol: Masking tuning times through pipelining in WDM optical networks. In Proceedings of INFOCOM '94, pages 1528-1535. IEEE, June 1994.

[14] G. R. Pieris and G. H. Sasaki. Scheduling trabsmissions in WDM broadcast-and-select networks. IEEE/ACM Transactions on Networking, 2(2):105-110, April 1994.

[15] A. Aggarwal, A. Bar-Noy, D. Coppersmith, R. Ramaswami, and B. Schieber. Efficient routing and scheduling algorithms for optical networks. Technical Report RC 18967, IBM Research Report, 1994.

[16] M. S. Borella and B. Mukherjee. Efficient scheduling of nonuniform packet traffic in a WDM/TDM local lightwave network with arbitrary transceiver tuning latencies. In Proceedings of INFOCOM'95, pages 129-136. IEEE, April 1995.

[17] T. Gonzalez and S. Sahni. Open shop scheduling to minimize finish time. Journal of the Association for Computing Machinery, 23(4):665-679, Oct 1976.

[18] E. Coffman, M. R. Garey, and D. S. Johnson. An application of bin-packing to multiprocessor scheduling. SIAM Journal of Computing, 7:1-17, Feb 1978.

[19] M. R. Garey, R. L. Graham, and D. S. Johnson. Performance guarantees for scheduling algorithms. Operations Research, 26:3-21, Jan 1978.

[20] M. R. Garey and D. S. Johnson. Computers and Intractability. W. H. Freeman and Co., New York, 1979. 


\section{A Proof that $O S T L$ is $\mathcal{N} \mathcal{P}$-complete for $C=2$ Channels}

We now show that $O S T L$ is $\mathcal{N} \mathcal{P}$-complete, even when the number of wavelengths $C=2$. As in [17], the proof uses a transformation from PARTITION, a well-known $\mathcal{N} \mathcal{P}$-complete problem [20] described below. However, our proof is substantially different, reflecting the fact that (a) the transformation is to an instance of $O S T L$ with $C=2$ channels, while the transformation in [17] is to an instance of $O S$ with $C=3$ channels, and (b) the tuning latency, $\Delta$, is now an influencing parameter.

Problem A.1 (PARTITION) Given a set $\mathcal{V}=\{1,2, \ldots, n\}$ with $w_{i}$ the weight of element $i$, and $W=\sum_{i=1}^{n} w_{i}$, is there a partition of $\mathcal{V}$ into two sets, $\mathcal{V}_{1}$ and $\mathcal{V}_{2}$, such that $\sum_{i \in \mathcal{V}_{1}} w_{i}=\sum_{j \in \mathcal{V}_{2}} w_{j}=$ $\frac{W}{2}$ ? (The $w_{i}$ 's may be assumed integer.)

Proof (of Theorem 4.1). It is easy to see that $O S T L$ is in the class $\mathcal{N P}$, since a nondeterministic algorithm need only (a) guess the optimal set of start slots $\left\{\tau_{i c}\right\}$ satisfying constraints (5), and (b) (6) and verify that the length of the schedule is less than or equal to $M$.

We now transform PARTITION to OSTL; note that it is sufficient to find a transformation for the case $C=2$. Let $\mathcal{V}=\{1,2, \ldots, n\}$ be the set of elements of weights $w_{i}, i=1, \ldots, n$, making up an arbitrary instance of PARTITION, and let $W=\sum_{i=1}^{n} w_{i}$. We construct an instance of $O S T L$ with $N=n+1$ stations, $C=2$ wavelengths, $\Delta=\frac{W^{2}}{2}, M=\frac{3 W^{2}}{2}+W$, and a collapsed traffic matrix, A, such that:

$$
\begin{gathered}
a_{i 1}=w_{i}, \quad a_{i 2}=(W+1) w_{i}, \quad i=1, \ldots, n \\
a_{n+1,1}=W^{2}, \quad a_{n+1,2}=0
\end{gathered}
$$

It is obvious that this transformation can be performed in polynomial time. We now show that a schedule of length $\frac{3 W^{2}}{2}+W$ exists for the above instance of $O S T L$ if and only if $\mathcal{V}$ has a partition. If $\mathcal{V}$ has a partition, $\mathcal{V}_{1}, \mathcal{V}_{2}$, then there is a schedule of length equal to $\frac{3 W^{2}}{2}+W$; one such schedule is shown in Figure 20 (the initial tuning period of $\Delta$ slots is not shown there). Conversely, if an $O S T L$ schedule of length $\frac{3 W^{2}}{2}+W$ exists, then $\mathcal{V}$ has a partition. This is shown in the following.

Let $\mathcal{S}=\left\{\tau_{i c}\right\}, i=1, \ldots, n+1, c=1,2$, be a schedule of length $\frac{3 W^{2}}{2}+W$. The first $\Delta=\frac{W^{2}}{2}$ slots are used for tuning of the transmitters to the first channel in their respective channel sequence, so let us consider the remaining $W^{2}+W$ slots. Also let our reference point (i.e., slot 1 ) be the first slot following these initial $\Delta$ slots. Since $\sum_{i=1}^{n} a_{i 2}=W^{2}+W$, all these slots of the schedule will contain a transmission by some source on channel $\lambda_{2}$. Now, the start slot, $\tau_{n+1,1}$, of station $n+1$ on channel 


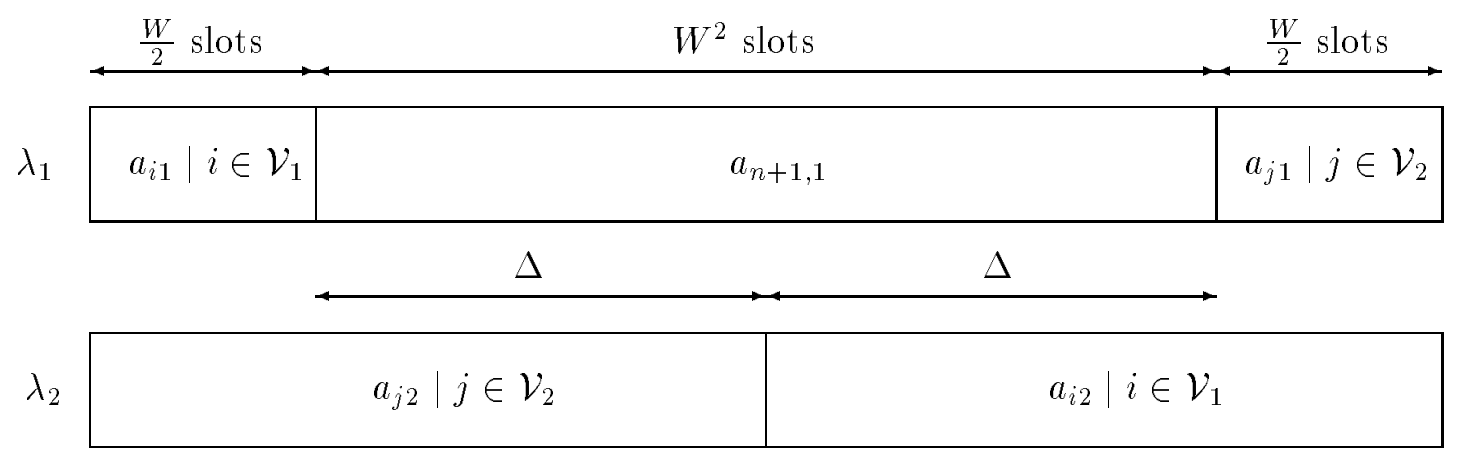

Figure 20: Optimum length schedule when $\mathcal{V}$ has a partition $\mathcal{V}_{1}, \mathcal{V}_{2}$ (the initial tuning period of $\Delta=\frac{W^{2}}{2}$ slots is not shown)

$\lambda_{1}$, must be $>1$. Otherwise, stations 1 through $n$ are all assigned to transmit on channel $\lambda_{1}$ in slots $W^{2}+1$ through $W^{2}+W$. But then, since $\Delta=\frac{W^{2}}{2}$, the hardware constraint (5) would not be satisfied for the station, say, $i$, assigned to transmit on channel $\lambda_{2}$ in slot $W^{2}+\frac{W}{2}$, contradicting the hypothesis that $\mathcal{S}$ is an admissible schedule. Similarly, it can be shown that $\tau_{n+1,1} \leq W$; in other words, station $n+1$ is not assigned to transmit in slots $W+1$ through $W^{2}+W$. As a result, stations 1 through $n$ are divided into two sets, $\mathcal{V}_{1}$ and $\mathcal{V}_{2}$, such that all stations in $\mathcal{V}_{1}$ (respectively, $\mathcal{V}_{2}$ ) have start slots on channel $\lambda_{1}$ less than $\tau_{n+1,1}$ (respectively, greater than or equal to $\tau_{n+1,1}+a_{n+1,1}$ ).

Let $W_{1}=\sum_{i \in \mathcal{V}_{1}} w_{i}$ and $W_{2}=\sum_{j \in \mathcal{V}_{2}} w_{j}$. Without loss of generality, let $W_{1} \geq W_{2}$; the case $W_{1} \leq W_{2}$ is handled similarly. Then, $W_{1}=\frac{W}{2}+\epsilon$, where $\epsilon \geq 0$. Since $\mathcal{S}$ satisfies the hardware constraints (5), we have that, for all $i \in \mathcal{V}_{1}: \tau_{i 1}+a_{i 1}+\Delta \leq \tau_{i 2}$. But $\tau_{i 1} \geq 1$ and $a_{i 1} \geq 1$ for all stations 1 through $n$, therefore $\tau_{i 2} \geq \Delta+2=\frac{W^{2}}{2}+2$, for all $i \in \mathcal{V}_{1}$, leaving a total of at most $\frac{W^{2}+2 W}{2}-1$ slots for transmissions by stations in $\mathcal{V}_{1}$ on $\lambda_{2}$. Also, the total number of slots allocated to stations in $\mathcal{V}_{1}$ for transmissions on channel $\lambda_{2}$ must be equal to $\sum_{i \in \mathcal{V}_{1}} a_{i 2}=(W+1)\left(\frac{W}{2}+\epsilon\right)$. Then,

$$
\frac{W^{2}+2 W}{2}-1 \geq(W+1)\left(\frac{W}{2}+e\right) \Rightarrow e \leq \frac{W-2}{2 W+2}<\frac{1}{2} \forall W>0
$$

Since $W_{1}=\frac{W}{2}+e$ must also be an integer, $\epsilon$ must be zero. In other words, $W_{1}=W_{2}=\frac{W}{2}$, and $\mathcal{V}_{1}, \mathcal{V}_{2}$ constitute a partition of $\mathcal{V}$ 


\section{B Proof of Theorem 5.1}

We now prove Theorem 5.1.

Proof (of Theorem 5.1). Let Sched(c) denote the frame of the schedule on channel $\lambda_{c}$ starting with the first slot in which transmitter 1 transmits on channel $\lambda_{c}$. Sched $(C+1)$ refers to the next frame on channel $\lambda_{1}$. Note that once the schedule length $M$ and the gaps $g_{i c}, i=1, \ldots, N-1$, are known, the gap $g_{N c}$ after the last transmitter is uniquely determined. Therefore we are not interested in the gaps that follow the last transmitter on each channel, and any reference to "gaps" in what follows does not include this last gap on each channel.

Let $O P T$ denote the optimal schedule length under the assumptions of Theorem 5.1. We will prove that $O P T \geq M$, hence proving that $O P T=M$. To do so, we trace through the algorithm as it computes $M$ and show that $O P T \geq M$ at every step of the algorithm.

That $O P T \geq M$ at the end of Step 2 is obvious, since the optimal can be no smaller than the lower bound. In Pass 1, all transmitters are assigned the earliest possible slots on each channel, and Step 9 makes sure that the schedule length is large enough so that each transmitter gets enough time to tune back to channel $\lambda_{1}$ after its transmission on channel $\lambda_{C}$ (in fact this is exactly what constraint (20) tries to capture). Therefore $O P T \geq M$ at the end of Pass 1 .

In Pass 2, channels as well as transmitters are processed in reverse order, and the algorithm tries to compact the gaps $g_{i c}, i=1, \ldots, N-1, c=2, \ldots C$, as much as possible. We show that once the gaps on a channel $\lambda_{c}$ have been compacted by Pass 2 of the algorithm above, it is not possible to compact them any further to reduce the schedule length, thus proving that $O P T \geq M$. The proof is by a two-level induction - the first on $c$ and the second on $i$ within the same channel $\lambda_{c}$. The induction proceeds by assuming that $\operatorname{Sched}(c+1)$ is optimal (meaning that the gaps on channel $\lambda_{c+1}$ cannot be compacted any further), and that transmitters $i+1 \ldots N$ are optimally scheduled on channel $\lambda_{c}$ (i.e., that the gaps $g_{i+1, c} \ldots g_{N-1, c}$ cannot be compacted any further; note that gap $g_{N c}$ is not considered), and then showing that the gap $g_{i c}$ cannot be compacted any more than what Pass 2 does. There are only 2 ways gap $g_{i c}$ can be compacted - either by moving the $a_{i c}$ slots to the right, or by moving slots $a_{j c}, j=i+1, \ldots, N$, to the left. But the $a_{i c}$ slots cannot be moved any more to the right (otherwise Step 12 would have done so), neither can slots $a_{j c}$ be moved any more to the left (otherwise Step 14 would have done so). Hence gap $g_{i c}$ is as compact as can be, and hence channel $\lambda_{c}$ is optimal by induction. To complete the induction proof, note that the inductive hypothesis holds for $c=C$, since $\operatorname{Sched}(C+1)$ is the same as the schedule on channel $\lambda_{1}$, which is optimal by assumption, as we only consider schedules in which channel $\lambda_{1}$ is idle only at the end of the frame (this will happen if at the end of the algorithm $M>\sum_{i=1}^{N} a_{i 1}$ ). 